Pacific

Journal of

Mathematics

GENERALIZED IMMERSIONS AND THE RANK OF THE SECOND FUNDAMENTAL FORM

Robert J. Fisher and H. TURner Laquer 


\title{
GENERALIZED IMMERSIONS AND THE RANK OF THE SECOND FUNDAMENTAL FORM
}

\author{
Robert J. FISHER AND H. TURNER LAQUER
}

To Hans Fischer and Raoul Bott

\begin{abstract}
Partial differential equations and differential geometry come together in the idea of a generalized immersion. This concept, defined by means of Grassmann bundles and contact forms, allows for "immersions" with "singularities." Sophus Lie's generalized solutions to partial differential equations are an important special case.

The classical second fundamental form has a natural generalization in the context of generalized immersions. The rank of the form is then meaningful. A constant rank assumption on the generalized second fundamental form leads to a natural foliation of the generalized immersion, at least when the ambient space is a space of constant curvature. Questions about the total geodesy and regularity of the foliation are also addressed.
\end{abstract}

\section{Introduction}

Our primary goal in this paper is to develop the idea of a generalized immersion and to understand the natural foliation of a generalized immersion that arises from a constant rank assumption on its generalized second fundamental form. Briefly, our main theorems are as follows: Theorem 1 deals with the natural developable foliation of a constant rank generalized immersion; Theorem 2 addresses the issue of the total geodesy of the leaves of the foliation; and Theorem 3 deals with the existence of local slices for the foliation in the vector space case.

Our definition of a generalized immersion is motivated by the geometrical approach to first order partial differential equations, as formulated by Cartan and Lie; see [Alekseevskij et al. 1991]. In this approach, a first order system of PDEs is expressed as a submanifold of the 1-jets, say

$$
\mathscr{E} \subset J^{1}\left(\mathbb{R}^{n}, \mathbb{R}^{\ell}\right)=\mathbb{R}^{n} \times \mathbb{R}^{\ell} \times L\left(\mathbb{R}^{n}, \mathbb{R}^{\ell}\right),
$$

MSC2000: primary 53C15, 53C42; secondary 35F20, 53C12, 58A30, $58 \mathrm{G} 30$.

Keywords: partial differential equation, generalized immersion, contact form, second fundamental form, connection, foliation, developable. 
and a function $f: \mathbb{R}^{n} \rightarrow \mathbb{R}^{\ell}$ is a solution to the system precisely when the prolongation of $f$ lies in $\mathscr{E}$, that is, $\{(x, f(x), D f(x))\} \subset \mathscr{E}$.

Next, the contact form is introduced. This is a natural $\mathbb{R}^{\ell}$-valued 1 -form on $J^{1}\left(\mathbb{R}^{n}, \mathbb{R}^{\ell}\right)$ that annihilates vectors tangent to prolongations. It is defined by

$$
\omega_{(x, z, A)}(h, k, B)=k-A \cdot h .
$$

In addition, a graphical submanifold $\{(x, f(x), A(x))\} \subset J^{1}\left(\mathbb{R}^{n}, \mathbb{R}^{\ell}\right)$ is annihilated by contact precisely when $A(x)=D f(x)$, for all $\mathrm{x}$, i.e., the graph is a prolongation. It is at this point that the theory broadens the meaning of "solution" by defining a generalized solution of a PDE to be an immersed $n$-dimensional submanifold of the equation manifold $\mathscr{E}$ that is annihilated by contact. The generalized solutions are interpreted as "singular" solutions to the PDE.

This is not entirely satisfying. On one hand, there is a real change in emphasis in going from prolongations of functions to immersions annihilated by contact. In particular, a prolongation has a canonical parametrization. On the other hand, when we allow arbitrary immersions, the jet formulation breaks down if the immersion is not locally the graph of a function. Finally, as geometers, we would also like to consider PDEs on arbitrary manifolds.

The ideas of contact and prolongation are essential in our formulation of a generalized immersion. Details appear in Section 1, but briefly, prolongation is a process that creates a generalized immersion from a classical immersion by including tangent map information. More precisely, if $\gamma^{0}: M \rightarrow \bar{M}$ is an immersion then the prolongation of $\gamma^{0}$ is the immersion $\gamma^{1}: M \rightarrow \mathrm{Gr}_{n}(T \bar{M})$ defined by

$$
\gamma^{1}(m)=\operatorname{Image}\left(\left.T \gamma^{0}\right|_{T_{m} M}\right),
$$

where $\pi: \operatorname{Gr}_{n}(T \bar{M}) \rightarrow \bar{M}$ is the Grassmann bundle of $n$-planes in the tangent bundle to $\bar{M}$. The Grassmann bundle plays a role analogous to the 1 -jets. In particular, there is a natural bundle-valued contact form on the Grassmann bundle that gives a way to understand vectors tangent to prolongations; see Lemma 1.1.

We now define a generalized immersion of $M$ in $\bar{M}$ to be an ordinary immersion of $M$ in $\operatorname{Gr}_{n}(T \bar{M})$ that is annihilated by contact. Technically, a classical immersion is not a generalized immersion. However, if $I(M, \bar{M})$ denotes the set of all immersions of a manifold $M$ into a manifold $\bar{M}$ and if $\operatorname{GI}(M, \bar{M})$ denotes the set of all generalized immersions of $M$ in $\bar{M}$, then prolongation defines a canonical injection

$$
I(M, \bar{M}) \rightarrow \mathrm{GI}(M, \bar{M}) .
$$

Although we do not address the issue of topologies on these spaces, the canonical injection allows for the understanding of generalized immersions as (possibly singular) limits of families of classical immersions. For example, the radius of 
a cylinder shrinks to zero or a family of hyperboloids degenerates into a cone. It is precisely the contact condition that makes the generalized immersions in $\mathrm{GI}(M, \bar{M})$ behave like (prolongations of) classical immersions of $M$ in $\bar{M}$. In particular, if we start with a generalized immersion $\gamma: M \rightarrow \operatorname{Gr}_{n}(T \bar{M})$ and let $\gamma^{0}: M \rightarrow \bar{M}$ be the point map $\pi \circ \gamma$, then $\gamma$ agrees with the prolongation of $\gamma^{0}$ at any point where $\gamma^{0}$ is an immersion.

Perhaps a word or two is in order about our term "generalized immersion." Although a generalized immersion is encoded by means of an ordinary immersion, it should not be thought of as a special type of immersion (as would be implied by the term "contact immersion"). Instead, generalized immersions represent an enlargement of the class of immersions. Moreover, our use of the term "generalized" is meant to reflect the analogy between generalized immersions and the idea of generalized solutions to partial differential equations, as defined by Lie.

Section 2 is devoted to the development of the generalized second fundamental form of a generalized immersion. Lemma 2.2 provides an important pointwise formula for the generalized second fundamental form. Its description requires some work with connections, specifically a connection in the Grassmann bundle, along with some local Grassmann manifold geometry. This section also introduces the reader to the full force of local computations using coordinate-free vector space computations. The papers [Fisher and Laquer 1999] and [Vilms 1967] can serve as introductions to some of these ideas.

Section 3 contains the proof of Theorem 1. In short, Theorem 1 states that a constant rank generalized immersion into a Riemannian manifold of constant curvature has a natural developable foliation (and conversely). First, by the rank of the generalized immersion at a point, we mean the rank of the generalized second fundamental form interpreted as a linear map; see (2-4). Because of the lower semicontinuity of rank, it is reasonable to assume that the rank is in fact constantit is always locally constant on an open dense subset. Secondly, the kernel of the generalized second fundamental form gives the involutive distribution needed to create the foliation. Thirdly, the idea of a developable foliation generalizes a classical developable surface, i.e., a ruled surface for which the unit normal is constant along rulings; see [Klingenberg 1978; Wu 1995].

Theorem 2 in Section 4 gives conditions on a constant rank generalized immersion that imply the leaves of the resulting foliation immerse as totally geodesic submanifolds. The symmetry of the ordinary second fundamental form is lost in the generalized setup. However, a partial symmetry remains and is exploited to study the interaction between the left and right kernels of the generalized second fundamental form. This interaction is then used to prove the total geodesy in three important cases.

In Section 5 we return to the setting of vector spaces, proving in Theorem 3 the 
existence of local cross-sections to the foliation in the case of constant-rank generalized immersions into vector spaces. This is useful since it guarantees that the resulting leaf space is a $T_{1}$-manifold. Finally, Section 6 contains several examples illustrating ideas from the paper.

\section{Generalized immersions}

Let $\bar{M}$ be a smooth Riemannian manifold of dimension $n+\ell$ and let

$$
\pi: \mathrm{Gr}_{n}(T \bar{M}) \rightarrow \bar{M}
$$

denote the Grassmann bundle of $n$-planes in the tangent bundle of $\bar{M}$. The pullback bundle $\pi^{*}(T \bar{M})$ has a canonical subbundle $\mathbb{U}$ of rank $n$ that is obtained as follows: let $P \in \mathrm{Gr}_{n}(T \bar{M})$. Then $P$ is an $n$-dimensional subspace of $T_{\pi(P)} \bar{M}$. Define

$$
\mathbb{U}=\left\{(P, h) \in \pi^{*}(T \bar{M}) \mid h \in P\right\},
$$

that is, the fiber at $P$ is $P$ itself. Commonly, $\mathbb{U}$ is referred to as the universal $n$-plane bundle over $\operatorname{Gr}_{n}(T \bar{M})$. Let

$$
\mathbb{Q}=\pi^{*}(T \bar{M}) / \mathbb{U}
$$

be the resulting quotient bundle. At a plane $P \in \mathrm{Gr}_{n}(T \bar{M})$, the fiber is the $\ell$-dimensional vector space

$$
\mathbb{Q}_{P}=\left(T_{\pi(P)} \bar{M}\right) / P \text {. }
$$

There is a canonical bundle-valued 1-form $\omega \in A^{1}\left(\operatorname{Gr}_{n}(T \bar{M}), \mathbb{Q}\right)$ defined by

$$
\omega_{P}(v)=T \pi(v)+P \quad \text { for } v \in T_{P} \operatorname{Gr}_{n}(T \bar{M}),
$$

i.e., $\omega_{P}(v)$ is the coset in $\mathbb{Q}_{P}$ represented by $T \pi(v)$. For reasons to be explained later, $\omega$ will be referred to as the contact form. Let $M$ be a smooth $n$-manifold. A generalized immersion of $M$ in $\bar{M}$ is an ordinary immersion $\gamma: M \rightarrow \operatorname{Gr}_{n}(T \bar{M})$ annihilated by contact, that is, for each $m \in M$ and each $h \in T_{m} M$,

$$
\omega_{\gamma(m)}(T \gamma(h))=0,
$$

or equivalently, $T \pi(T \gamma(h)) \in \gamma(m)$.

Let $\gamma^{0}: M \rightarrow \bar{M}$ be an immersion. Then the tangent map of $\gamma^{0}$ defines a generalized immersion in a canonical manner: for each $m \in M$, the restriction of the tangent map $T \gamma^{0}: T_{m} M \rightarrow T_{\gamma^{0}(m)} \bar{M}$ is an injective linear map. Thus the image of $T \gamma^{0}$ at $m$ gives a point in $\operatorname{Gr}_{n}\left(T_{\gamma^{0}(m)} \bar{M}\right)$ and we define $\gamma^{1}: M \rightarrow \operatorname{Gr}_{n}(T \bar{M})$ by

$$
\gamma^{1}(m)=\operatorname{Image}\left(\left.T \gamma^{0}\right|_{T_{m} M}\right) \text {. }
$$


We call $\gamma^{1}$ the prolongation (or 1-jet prolongation; see [Binz et al. 1988]) of $\gamma^{0}$. Note that Jensen and Rigoli [1989] use the term "Gauss map" for the prolongation, even though they recognize that it is a "quasi-generalization" of the classical Gauss map. Our preference is to use the term "Gauss map" only for the principal part of the prolongation in the vector space case.

Next, the equation $\gamma^{0}=\pi \circ \gamma^{1}$ implies that for each $h \in T_{m} M$,

$$
T \gamma^{0}(h)=T \pi \circ T \gamma^{1}(h) \in \gamma^{1}(m) .
$$

Thus, $\omega_{\gamma^{1}(m)}\left(T \gamma^{1}(h)\right)=0$ as expected. Conversely, let $\gamma$ be a generalized immersion such that $\gamma^{0}=\pi \circ \gamma$ is a classical immersion. Then $\gamma$ is the prolongation of $\gamma^{0}$ by the following argument: For each $h \in T_{m} M$, we have $T \pi(T \gamma(h)) \in \gamma(m)$ since the immersion $\gamma$ is annihilated by contact. But $T \gamma^{0}(h)=T \pi(T \gamma(h))$. So by the injectivity of $\left.T \gamma^{0}\right|_{T_{m} M}$, it follows that

$$
\text { Image }\left(\left.T \gamma^{0}\right|_{T_{m} M}\right)=\gamma(m) \text {. }
$$

More generally, suppose we start with a smooth mapping $\gamma^{0}: M \rightarrow \bar{M}$, not necessarily an immersion. Let $M_{0} \subseteq M$ be the set of all nonsingular points, i.e., all points where the tangent mapping is injective. If $\gamma: M \rightarrow \mathrm{Gr}_{n}(T \bar{M})$ is any lift of $\gamma^{0}$ to a generalized immersion of $M$ in $\bar{M}$ then, as before, $\gamma(m)=\operatorname{Image}\left(\left.T \gamma^{0}\right|_{T_{m} M}\right)$ for $m \in M_{0}$. Next, suppose $M_{0}$ is dense in $M$. By continuity, there can be at most one continuous extension of $\gamma$ from $M_{0}$ to $M$. Thus there can be at most one generalized immersion $\gamma: M \rightarrow \mathrm{Gr}_{n}(T \bar{M})$ with $\gamma^{0}=\pi \circ \gamma$. Moreover, if the unique extension is smooth then continuity and the assumption that $M_{0}$ is dense in $M$ will imply that the extension is annihilated by contact. For example, this principle shows how to create a generalized immersion from a cone (including the cone point) or from a tangentially developed surface (see Example 6.1).

The contact form $\omega$ is canonically interpreted as a vector bundle morphism

$$
\omega: T \mathrm{Gr}_{n}(T \bar{M}) \rightarrow \mathbb{Q} .
$$

Because $\pi: \operatorname{Gr}_{n}(T \bar{M}) \rightarrow \bar{M}$ is a submersion, it follows that $\omega$ is an epimorphism of vector bundles and thus $\operatorname{ker} \omega$ is a vector subbundle of $T \mathrm{Gr}_{n}(T \bar{M})$, i.e., a distribution on $\mathrm{Gr}_{n}(T \bar{M})$.

Let $v \in \operatorname{ker} \omega_{P}$ for some $P \in \mathrm{Gr}_{n}(T \bar{M})$. Since

$$
\operatorname{ker} \omega \rightarrow \mathrm{Gr}_{n}(T \bar{M})
$$

is a vector bundle, there is a smooth section $X$ of $\operatorname{ker} \omega$ such that $X(P)=v$. The existence and uniqueness theorem of ODEs then implies that there is a smooth curve $P(t)$ of $n$-planes such that $P(0)=P$ and $X(P(t))=[s \mapsto P(t+s)]$. Note, if $c(s)$ is a curve in a manifold then the notation $[s \mapsto c(s)]$ or just $[c(s)]$ denotes the vector tangent to $c$ at $s=0$. Thus for each contact vector $v \in \operatorname{ker} \omega_{P}$, there is 
an integral curve $P(t)$ of the distribution $\operatorname{ker} \omega$ with $[P(t)]=v$. We will call such a curve in $\mathrm{Gr}_{n}(T \bar{M})$ a "contact curve" or a "spine of $n$-planes."

An alternative view of contact vectors is given by the Cartan distribution. Formally, this is the subbundle $\mathscr{C}$ of $T \mathrm{Gr}_{n}(T \bar{M})$ whose fiber at $P \in \mathrm{Gr}_{n}(T \bar{M})$ is the span of all vectors tangent to prolongations through $P$. The following lemma shows $\mathscr{C}$ is a distribution and is our reason for referring to $\omega$ as the contact form.

\section{Lemma 1.1. The kernel of $\omega$ is the Cartan distribution.}

Proof. We have seen that $\mathscr{C} \subset \operatorname{ker} \omega$. For the reverse containment, we will argue that nonvertical contact vectors, that is vectors $v \in \operatorname{ker} \omega$ such that $T \pi(v) \neq 0$, are tangent to prolongations. From this it follows that vertical vectors are in $\mathscr{b}$, because any vertical vector is the difference of two nonvertical contact vectors.

Let $v$ be a nonvertical contact vector based at an $n$-plane $P_{0}$. We will produce an immersion $\gamma^{0}: M \rightarrow \bar{M}$ of a smooth $n$-manifold and a point $m_{0} \in M$ satisfying the following conditions:

(1) $\gamma^{0}\left(m_{0}\right)=\pi\left(P_{0}\right)$.

(2) The image of the tangent mapping $T \gamma^{0}$ at $m_{0}$ is $P_{0}$.

(3) $v$ is tangent to the prolongation of $\gamma^{0}$ at $P_{0}$.

Let $P: I=(-\epsilon, \epsilon) \rightarrow \mathrm{Gr}_{n}(T \bar{M})$ be a spine of planes representing the contact vector $v$, let $c(t)=\pi(P(t))$ and let $c^{\prime}(t)=[s \mapsto c(t+s)]$. Because $T \pi(v)=c^{\prime}(0) \neq 0$, we can assume that $c^{\prime}(t) \neq 0$, for all $t \in I$. By the spine condition, $c^{\prime}(t) \in P(t)$ for all $t \in I$, so the pullback bundle $P^{*}(\mathbb{U}) \rightarrow I$ has a natural line subbundle $\mathbb{L} \rightarrow I$ defined by $\mathbb{L}_{t}=\mathbb{R} \cdot c^{\prime}(t)$. Let $\mathbb{M} \subset P^{*}(\mathbb{U})$ be any rank $(n-1)$-subbundle that is complementary to $\mathbb{L}$. The eventual domain for the immersion $\gamma^{0}$ will be an open neighborhood $M$ in $\mathbb{M}$ of a point $m_{0}$ in the zero section. Keeping in mind that $P^{*}(\mathbb{U})=\left\{(t, h) \in I \times \mathbb{U} \mid h \in P(t) \subset T_{c(t)} \bar{M}\right\}$, define $\gamma^{0}: M \rightarrow \bar{M}$ by

$$
\gamma^{0}(t, h)=\exp (h)
$$

Here exp is the exponential map on $\bar{M}$ as determined by the choice of a Riemannian metric or spray on $\bar{M}$; see [Dieudonné 1974, (18.4)] and [Fisher and Laquer 1999]. By choosing $I$ and $M$ appropriately, $h$ will be sufficiently close to zero so that $\exp (h)$ is defined whenever $(t, h) \in M$.

The key part of the argument is to compute the tangent map of $\gamma^{0}$ along the zero section in $M$ showing that

$$
P(t)=\text { Image }\left(\left.T \gamma^{0}\right|_{T_{(t, 0)} M}\right) .
$$

This implies that $\gamma^{0}$ is an immersion in some neighborhood of $m_{0}=(0,0) \in M$ and moreover, that the curve $P(t)$ lies in the prolongation of $\gamma^{0}$. Thus $v$ is tangent to the prolongation of $\gamma^{0}$. 
The tangent space $T_{(t, 0)} M$ has a natural splitting into vertical vectors and vectors tangent to the zero section. The former are represented by represented by curves $s \mapsto(t, s \cdot x)$ for $x \in \mathbb{M}_{t}$, while the latter are represented by curves $s \mapsto(t+s, 0) \in M$. By standard properties of the exponential mapping, see [Dieudonné 1974, (18.4.5)],

$$
T \gamma^{0}[s \mapsto(t+s, 0)]=\left[s \mapsto \gamma^{0}(t+s, 0)\right]=[s \mapsto c(t+s)]=c^{\prime}(t)
$$

and

$$
T \gamma^{0}[s \mapsto(t, s \cdot x)]=[s \mapsto \exp (s \cdot x)]=x .
$$

Thus the image of $T \gamma^{0}$ at $(t, 0)$ is given by the span of $c^{\prime}(t)$ and $\mathbb{M}_{t}$. This gives (1-2) and completes the proof of the lemma.

\section{The generalized second fundamental form}

Let $\bar{M}$ be a smooth Riemannian manifold of dimension $n+\ell$, and consider a generalized immersion $\gamma: M \rightarrow \operatorname{Gr}_{n}(T \bar{M})$. Our purpose in this section is to develop the concept of the generalized second fundamental form of $M$ in $\bar{M}$. We maintain the notation and terminology of the previous section.

The Riemannian metric on $\bar{M}$ gives a splitting

$$
\pi^{*}(T \bar{M})=\mathbb{U} \oplus \mathbb{U}^{\perp} .
$$

Next let $\gamma^{0}=\pi \circ \gamma$ and let $\mathbb{B}=\left(\gamma^{0}\right)^{*}(T \bar{M})$. From (2-1), it follows that $\mathbb{B}$ splits:

$$
\mathbb{B}=\mathrm{GT}(M) \oplus \mathrm{GN}(M)
$$

where $\mathrm{GT}(M)=\gamma^{*}(\mathbb{U})$ and $\mathrm{GN}(M)=\gamma^{*}\left(\mathbb{U}^{\perp}\right)$. We will refer to $\mathrm{GT}(M)$ and $\mathrm{GN}(M)$ as the generalized tangent and generalized normal bundles of the generalized immersion. Let $\bar{\nabla}$ denote the pullback to $\mathbb{B}$ of the Levi-Civita connection on $\bar{M}$. For each vector field $X \in \mathfrak{X}(M)$ and each section $\sigma \in \Gamma(\mathrm{GT}(M))$, we use (2-2) to write

$$
\bar{\nabla}_{X} \sigma=\tan \left(\bar{\nabla}_{X} \sigma\right)+\operatorname{nor}\left(\bar{\nabla}_{X} \sigma\right) .
$$

Then the generalized tangential component

$$
\nabla_{X} \sigma=\tan \left(\bar{\nabla}_{X} \sigma\right)
$$

defines a connection in $\mathrm{GT}(M)$ while the generalized normal component

$$
\mathrm{II}(X, \sigma)=\operatorname{nor}\left(\bar{\nabla}_{X} \sigma\right)
$$

defines a $\mathrm{GN}(M)$-valued tensor which we will call the generalized second fundamental form. Similarly, for each $X \in \mathfrak{X}(M)$ and each $\eta \in \Gamma(\mathrm{GN}(M))$,

$$
\nabla_{X}^{\perp} \eta=\operatorname{nor}\left(\bar{\nabla}_{X} \eta\right)
$$


defines a connection in $\mathrm{GN}(M)$ while

$$
\mathrm{II}^{\perp}(X, \eta)=\tan \left(\bar{\nabla}_{X} \eta\right)
$$

is a $\mathrm{GT}(M)$-valued tensor.

When $M$ is a classically immersed submanifold of $\bar{M}$, the classical second fundamental form II $^{M}$ is the generalized second fundamental form II of the prolongation. Recall that the classical second fundamental form of an immersion $\gamma^{0}: M \rightarrow \bar{M}$ is obtained from the splitting

$$
\left(\gamma^{0}\right)^{*}(T \bar{M})=T \gamma^{0}(T M) \oplus T \gamma^{0}(T M)^{\perp} .
$$

Let $\bar{\nabla}$ denote the pullback of the Levi-Civita connection on $\bar{M}$. The splitting (2-3) defines the induced connection $\nabla^{M}$ in $T \gamma^{0}(T M)$ and the corresponding second fundamental form II $^{M}$ by

$$
\bar{\nabla}_{X} \sigma=\nabla_{X}^{M} \sigma+\Pi^{M}(X, \sigma)
$$

for all $X \in \mathfrak{X}(M)$ and $\sigma \in \Gamma\left(T \gamma^{0}(T M)\right)$. Let $\gamma^{1}: M \rightarrow \operatorname{Gr}_{n}(T \bar{M})$ be the prolongation of $\gamma^{0}$. By way of the canonical identification between $\left(\gamma^{1}\right)^{*}\left(\pi^{*}(T \bar{M})\right)$ and $\left(\gamma^{0}\right)^{*}(T \bar{M}),(2-2)$ and (2-3) are the same splitting; thus $\nabla=\nabla^{M}$ and $\mathrm{II}=\mathrm{II}^{M}$.

For a generalized immersion $\gamma$ with $\gamma^{0}=\pi \circ \gamma$, the splitting in (2-2) is always defined. On the other hand, the splitting in (2-3) is defined if and only if $\gamma^{0}$ is a subimmersion. Indeed, if $\gamma^{0}$ is not a subimmersion, then $T \gamma^{0}(T M)$ is not a bundle, although the splitting

$$
\mathbb{B}_{m}=T \gamma^{0}\left(T_{m} M\right) \oplus T \gamma^{0}\left(T_{m} M\right)^{\perp}
$$

is defined for each $m \in M$. Moreover, when (2-3) is a bundle splitting, (2-2) and (2-3) agree if and only if $\gamma^{0}$ is an immersion. Example 6.2 shows that the two splittings can be distinct.

For each $m \in M$ the tensor II is viewed canonically as a linear map

$$
\mathrm{II}_{m}: T_{m} M \rightarrow L\left(\mathrm{GT}(M)_{m}, \mathrm{GN}(M)_{m}\right) .
$$

By the kernel of $\mathrm{II}_{m}$, we will always mean the following:

$$
\text { ker } \mathrm{II}_{m}=\left\{h \in T_{m} M \mid \mathrm{I}_{m}(h, \sigma)=0 \quad \forall \sigma \in \mathrm{GT}(M)_{m}\right\} .
$$

We will also be discussing the right kernel of $\Pi_{m}$, which is defined by

$$
R \text { ker } \Pi_{m}=\left\{\sigma \in \operatorname{GT}(M)_{m} \mid I_{m}(h, \sigma)=0 \quad \forall h \in T_{m} M\right\} .
$$

The generalized immersion is said to have rank $k$ if and only if the rank of the linear mapping $\mathbb{I}_{m}$ in (2-4) is equal to $k$ for all $m \in M$. Under the constant rank assumption, ker II defines a subbundle of $T M$ of rank $n-k$, i.e., a distribution on 
M. By the lower semicontinuity of rank [Dieudonné 1974, (18.10.1)], the rank of $\mathrm{II}_{m}$ is always locally constant on an open dense subset of $M$.

The connection form in $\mathbf{G r}_{\boldsymbol{n}}(\boldsymbol{T} \overline{\boldsymbol{M}})$. Following [Dieudonné 1972, (17.16.3)] and [Fisher and Laquer 1999, (§3)], let

$$
C: T \bar{M} \oplus T \bar{M} \rightarrow T(T \bar{M})
$$

be a connection in the tangent bundle to $\bar{M}$. Commonly, $C(h, x)$ is called the horizontal lift of $h$ that is based at $x$.

The Grassmann bundle is an associated fiber bundle of the frame bundle of $M$, so that a connection $C$ induces both a horizontal lift $\hat{C}$ and a connection form $\theta$ in $\mathrm{Gr}_{n}(T \bar{M})$. Both are described naturally in terms of parallel translation.

The horizontal lift $\hat{C}: \pi^{*}(T \bar{M}) \rightarrow T \mathrm{Gr}_{n}(T \bar{M})$ is defined by

$$
\hat{C}(P, h)=\left[\tau_{t}(P)\right],
$$

where $\tau_{t}(P)$ is the curve of $n$-planes obtained by parallel translation of $P$ along any curve $m(t)$ representing the tangent vector $h$. Since parallel translation is an isomorphism, the translation of $P$ as a set is again an $n$-plane.

Let $\mathbb{V}=\operatorname{ker} T \pi$, the bundle of vertical tangent vectors to $\operatorname{Gr}_{n}(T \bar{M})$. The connection form $\theta: T \mathrm{Gr}_{n}(T \bar{M}) \rightarrow \mathbb{V}$ is given by

$$
\theta(v)=v-\hat{C}\left(\pi_{\mathrm{Gr}}(v), T \pi \cdot v\right)=\left[\tau_{t}^{-1}(P(t))\right]
$$

where $v=[P(t)]$, i.e., the tangent vector represented by the curve $\tau_{t}^{-1}(P(t))$.

A more detailed discussion of $\hat{C}$ and $\theta$ in the general context of fiber bundles associated to a principal bundle is found in Chapter 20 of [Dieudonné 1974], and specifically problem 20.5.1.

Vertical vectors. Let $P \in \operatorname{Gr}_{n}(T \bar{M})$ and let $\bar{m}=\pi(P)$. The vertical space $\mathbb{V}_{P}$ is the tangent space at $P$ to the fiber $\pi^{-1}(\bar{m})=\operatorname{Gr}_{n}\left(T_{\bar{m}} \bar{M}\right)$, so that vertical vectors at a point are just tangent vectors to a single Grassmann manifold. Our immediate discussion will be in those terms. Let $E$ be a vector space of dimension $n+\ell$ and let $\mathrm{Gr}_{n}(E)$ denote the Grassmann manifold of all $n$-dimensional subspaces of $E$. A direct sum decomposition $E=P \oplus Q$ of $E$ gives rise to two natural projection maps $\operatorname{Proj}_{Q}^{P}: E \rightarrow E$ and $\operatorname{Proj}_{P}^{Q}: E \rightarrow E$, where $\operatorname{Proj}_{Q}^{P}$ is projection onto $Q$ parallel to (or with kernel) $P$. Using cosets, the projection is given by

$$
\operatorname{Proj}_{Q}^{P}(x)=(x+P) \cap Q .
$$

In other words, the intersection of the coset of $P$ through $x$ with $Q$ allows one to visualize the linear map $\operatorname{Proj}_{Q}^{P}$ as moving $x$ parallel to $P$ until it intersects $Q$.

Fix an inner product $\langle\cdot, \cdot\rangle$ on $E$. Let $P \in \operatorname{Gr}_{n}(E)$ and let $L\left(P, P^{\perp}\right)$ denote the vector space of linear maps $A: P \rightarrow P^{\perp}$. The canonical chart centered at $P$ is the 
set $U_{P}=\left\{H \in \operatorname{Gr}_{n}(E) \mid E=H \oplus P^{\perp}\right\}$ along with the mapping $\varphi_{P}: U_{P} \rightarrow L\left(P, P^{\perp}\right)$ defined as follows:

$$
\varphi_{P}(H)=A \quad \text { if and only if } \quad A x=-\left((x+H) \cap P^{\perp}\right) \text { for all } x \in P .
$$

In other words, $\varphi_{P}(H)=-\left.\operatorname{Proj}_{P \perp}^{H}\right|_{P}$. The inverse of $\varphi_{P}$ is the function

$$
\varphi_{P}^{-1}(A)=\{x+A x \mid x \in P\},
$$

that is, $\varphi_{P}^{-1}(A)$ is the graph of $A$ relative to the direct sum $E=P \oplus P^{\perp}$. We let $P_{A}$ denote $\varphi_{P}^{-1}(A)$.

The natural map Perp: $\mathrm{Gr}_{n}(E) \rightarrow \mathrm{Gr}_{\ell}(E)$ sending $P$ to $P^{\perp}$ is a diffeomorphism. To understand the canonical local form of Perp at $P \in \mathrm{Gr}_{n}(E)$, consider the standard charts $\left(U_{P}, \varphi_{P}\right)$ and $\left(U_{P^{\perp}}, \varphi_{P \perp}\right)$. It is routinely argued that Perp restricts to a bijection between $U_{P}$ and $U_{P^{\perp}}$. Next, let $A \in L\left(P, P^{\perp}\right)$. The transpose of $A$ relative to the inner product is the linear map ${ }^{t} A: P^{\perp} \rightarrow P$ that is defined by the equation

$$
\langle A x, y\rangle=\left\langle x,{ }^{t} A y\right\rangle
$$

for all $x \in P, y \in P^{\perp}$. In particular, for all $x \in P, y \in P^{\perp}$,

$$
\left\langle x+A x, y-{ }^{t} A y\right\rangle=\langle A x, y\rangle-\left\langle x,{ }^{t} A y\right\rangle=0 .
$$

From this it follows immediately that

$$
P_{A}^{\perp}=\left\{y-{ }^{t} A y \mid y \in P^{\perp}\right\} .
$$

Thus the local form of Perp is the linear isomorphism

$$
\varphi_{P \perp} \circ \operatorname{Perp} \circ \varphi_{P}^{-1}: L\left(P, P^{\perp}\right) \rightarrow L\left(P^{\perp}, P\right)
$$

given by

$$
\varphi_{P \perp} \circ \operatorname{Perp} \circ \varphi_{P}^{-1}(A)=-{ }^{t} A .
$$

Implicit in this discussion is the equality ${ }^{t} A=\left.\operatorname{Proj}_{P}^{P_{A}^{\perp}}\right|_{P^{\perp}}$. So geometrically, ${ }^{t} A$ moves a vector $y \in P^{\perp}$ parallel to $P_{A}^{\perp}$ until it intersects $P$ (see Figure 1).

Next let

$$
\psi_{P}=T \varphi_{P}: T_{P}\left(\operatorname{Gr}_{n}(E)\right) \rightarrow T_{0}\left(L\left(P, P^{\perp}\right)\right) \cong L\left(P, P^{\perp}\right) .
$$

Given a curve $P(t)$ that represents a tangent vector $v \in T_{P}\left(\operatorname{Gr}_{n}(E)\right)$, we have

$$
\psi_{P}(v)=\left(\varphi_{P} \circ P\right)^{\prime}(0) .
$$

For each $t, E$ splits as $P(t) \oplus P(t)^{\perp}$. Let

$$
\operatorname{Pr}_{t}=\operatorname{Proj}_{P(t)}^{P(t)^{\perp}} \quad \text { and } \quad \operatorname{Pr}_{t}^{\perp}=\operatorname{Proj}_{P(t)^{\perp}}^{P(t)}
$$




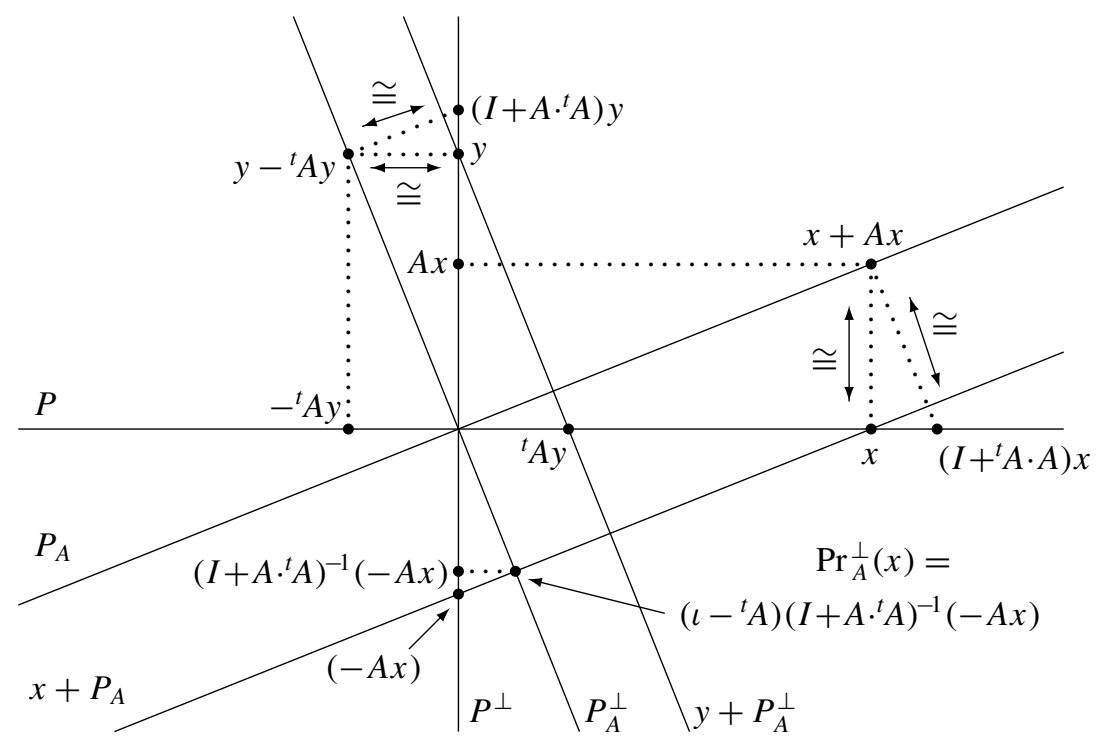

Figure 1. Projections determined by a local chart on $\mathrm{Gr}_{n}(E)$.

denote the corresponding projection maps determined by the direct sum, both viewed as curves in the vector space $L(E, E)$.

Lemma 2.1. $\psi_{P}([P(t)])=-\left(\left.\frac{d}{d t}\right|_{t=0} \operatorname{Pr}_{t}^{\perp}\right)$ where the right hand side is interpreted as an element of $L\left(P, P^{\perp}\right)$ by restriction.

Proof. To simplify notation, set $\alpha_{t}=\operatorname{Pr}_{t}^{\perp}$. The following argument shows that $\left.\frac{d}{d t}\right|_{t=0} \alpha_{t}$ maps $P$ into $P^{\perp}$, and so by restricting to $P,\left.\frac{d}{d t}\right|_{t=0} \alpha_{t}$ defines an element of $L\left(P, P^{\perp}\right)$. Let $h, k \in P$. Because $\left\langle\alpha_{t}(h), k\right\rangle=\left\langle\alpha_{t}(h), \alpha_{t}(k)\right\rangle$, it follows that

$$
\begin{aligned}
\left\langle\alpha_{0}^{\prime}(h), k\right\rangle & =\left.\frac{d}{d t}\right|_{t=0}\left\langle\alpha_{t}(h), k\right\rangle=\left.\frac{d}{d t}\right|_{t=0}\left\langle\alpha_{t}(h), \alpha_{t}(k)\right\rangle \\
& =\left\langle\alpha_{0}^{\prime}(h), 0\right\rangle+\left\langle 0, \alpha_{0}^{\prime}(k)\right\rangle=0 .
\end{aligned}
$$

Next, the linear mappings $\left(I+A \cdot{ }^{t} A\right): P^{\perp} \rightarrow P^{\perp}$ and $\left(I+{ }^{t} A \cdot A\right): P \rightarrow P$ are invertible because each is a composition of invertible linear maps. Explicitly,

$$
\left(I+A \cdot{ }^{t} A\right)=\left.\operatorname{Proj}_{P^{\perp}}^{P_{A}}\right|_{P_{A}^{\perp}} \circ\left(\iota-{ }^{t} A\right) \quad \text { and } \quad\left(I+{ }^{t} A \cdot A\right)=\left.\operatorname{Proj}_{P} P_{A}^{\perp}\right|_{P_{A}} \circ(\iota+A)
$$

where

$$
\left(\iota-{ }^{t} A\right)=\left.\operatorname{Proj}_{P_{A}^{\perp}}^{P}\right|_{P^{\perp}} \quad \text { and } \quad(\iota+A)=\left.\operatorname{Proj}_{P_{A}}^{P^{\perp}}\right|_{P} .
$$

The upper portion of Figure 1 provides a visual understanding of the first equation, while the second equation is expressed in the right portion of Figure 1. 
Supported by the lower portion of Figure 1, the previous paragraph implies routinely that the $P_{A}$ and $P_{A}^{\perp}$ components of $x \in P$ are

$$
\operatorname{Pr}_{A}(x)=(\iota+A)\left(I+{ }^{t} A \cdot A\right)^{-1}(x) \in P_{A}
$$

and

$$
\operatorname{Pr}_{A}^{\perp}(x)=\left(\iota-{ }^{t} A\right)\left(I+A \cdot{ }^{t} A\right)^{-1}(-A \cdot x) \in P_{A}^{\perp} .
$$

Set $A_{t}=\varphi_{P}(P(t))$. From above, it follows that for each $x \in P$

$$
\alpha_{t}(x)=-\left(\iota-{ }^{t} A_{t}\right)\left(I+A_{t} \cdot{ }^{t} A_{t}\right)^{-1}\left(A_{t} x\right) .
$$

So, since $A_{0}=0,{ }^{t} A_{0}=0$, and $A_{0}^{\prime}=\left(\varphi_{P} \circ P\right)^{\prime}(0)$, it follows that

$$
\left(\left.\frac{d}{d t}\right|_{t=0} \alpha_{t}\right)(x)=\left(\left.\frac{d}{d t}\right|_{t=0} \alpha_{t}(x)\right)=-A_{0}^{\prime} \cdot x=-\psi_{P}([P(t)])(x) .
$$

We will now use Lemma 2.1 to establish a formula for the generalized second fundamental form.

Lemma 2.2. At a point $m \in M, \mathrm{I}_{m}: T_{m} M \times \mathrm{GT}(M)_{m} \rightarrow \mathrm{GN}(M)_{m}$ is given by

$$
\mathbf{I}_{m}(h, \sigma)=\left(\psi_{\gamma(m)} \circ \theta \circ T \gamma(h)\right) \cdot \sigma .
$$

Proof. Clearly, the formula holds for $h=0$. So assume that $h \neq 0$ and that $m(t)$ is a curve representing $h$. Next, let $\bar{m}(t)=\left(\gamma^{0} \circ m\right)(t)$ and let

$$
\tau_{t}: T_{\bar{m}(0)} \bar{M} \rightarrow T_{\bar{m}(t)} \bar{M}
$$

denote the parallel translation in $\bar{M}$ along $\bar{m}(t)$. Set $\bar{P}(t)=(\gamma \circ m)(t)$ and

$$
P(t)=\tau_{t}^{-1}(\bar{P}(t)) .
$$

Then by (2-6), the curve $P(t)$ represents the vertical vector $\theta(T \gamma(h))$ based at the $n$-plane $P=P(0)=\gamma(m)$. There are two curves of projection maps, namely,

$$
\overline{\operatorname{Pr}}_{t}^{\perp}: T_{\bar{m}(t)} \bar{M}=\bar{P}(t) \oplus \bar{P}(t)^{\perp} \rightarrow \bar{P}(t)^{\perp}
$$

and

$$
\operatorname{Pr}_{t}^{\perp}: T_{\bar{m}(0)} \bar{M}=P(t) \oplus P(t)^{\perp} \rightarrow P(t)^{\perp} .
$$

Also present are the companion curves $\overline{\operatorname{Pr}}_{t}=I-\overline{\operatorname{Pr}}_{t}^{\perp}$ and $\operatorname{Pr}_{t}=I-\operatorname{Pr}{ }_{t}^{\perp}$. As before, these projections are viewed as endomorphisms of the appropriate spaces. By definition,

$$
\mathrm{II}_{m}(h, \sigma)=\operatorname{nor}\left(\bar{\nabla}_{h} \sigma\right)=\operatorname{Pr}_{0}^{\perp}\left(\left.\frac{d}{d t}\right|_{t=0} \tau_{t}^{-1} \sigma(m(t))\right),
$$

where $\sigma$ represents some extension of the vector $\sigma$ to a local section of $\mathrm{GT}(M)$. There is however a natural extension of $\sigma$, namely, one whose values along $m(t)$ are

$$
\overline{\operatorname{Pr}}_{t}\left(\tau_{t}(\sigma)\right) \in \bar{P}(t)=\mathrm{GT}(M)_{m(t)}
$$


Thus,

$$
\mathrm{II}_{m}(h, \sigma)=\operatorname{Pr}_{0}^{\perp}\left(\left.\frac{d}{d t}\right|_{t=0}\left(\tau_{t}^{-1} \circ \overline{\operatorname{Pr}}_{t} \circ \tau_{t}\right) \sigma\right) .
$$

On the other hand, because the parallel translation on $\bar{M}$ is an isometry,

$$
P(t)^{\perp}=\tau_{t}^{-1}\left(\bar{P}(t)^{\perp}\right)
$$

and consequently, the following diagram commutes:

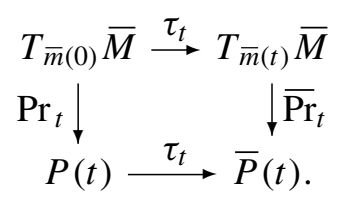

Thus, $\operatorname{Pr}_{t}=\tau_{t}^{-1} \circ \overline{\operatorname{Pr}}_{t} \circ \tau_{t}$. Therefore by Lemma 2.1 and (2-6),

$$
\begin{aligned}
\mathrm{II}_{m}(h, \sigma) & =\operatorname{Pr}_{0}^{\perp}\left(\left.\frac{d}{d t}\right|_{t=0} \operatorname{Pr}_{t}(\sigma)\right)=\operatorname{Pr}_{0}^{\perp}\left(\left.\frac{d}{d t}\right|_{t=0}\left(\sigma-\operatorname{Pr}_{t}^{\perp}(\sigma)\right)\right) \\
& =-\operatorname{Pr}_{0}^{\perp}\left(\left.\frac{d}{d t}\right|_{t=0} \operatorname{Pr}_{t}^{\perp}(\sigma)\right)=\psi_{\gamma(m)}([P(t)]) \cdot \sigma \\
& =\psi_{\gamma(m)}(\theta(T \gamma(h))) \cdot \sigma .
\end{aligned}
$$

\section{Developable generalized immersions}

A generalized immersion of $M$ in $\bar{M}$ is defined to be $(n-k)$-developable if and only if the following properties hold:

(1) $M$ has a foliation, written $M=\bigcup_{\alpha} \mathscr{L}_{\alpha}$, where $\operatorname{dim}\left(\mathscr{L}_{\alpha}\right)=n-k$.

(2) The restriction $\gamma: \mathscr{L}_{\alpha} \rightarrow \mathrm{Gr}_{n}(T \bar{M})$ is a horizontal immersion.

From property $2, \gamma\left(\mathscr{L}_{\alpha}\right)$ is transverse to the bundle projection, and hence $\gamma^{0}=\pi \circ \gamma$ restricts to an immersion of $\mathscr{L}_{\alpha}$ into $\bar{M}$ for each $\alpha$. We set $\overline{\mathscr{L}}_{\alpha}=\gamma^{0}\left(\mathscr{L}_{\alpha}\right)$. It is of interest to know whether or not the immersed leaves are totally geodesic submanifolds of $\bar{M}$. This issue is addressed by Theorem 2 in the following section and further ahead in the examples.

For us, a developable generalized immersion is a generalization of what is classically referred to as a "developable surface" in $\mathbb{R}^{3}$, that is, a ruled surface where the unit normal is constant along each ruling; see [Klingenberg 1978; Wu 1995]. We next state and prove the main theorem.

Theorem 1. Let $\bar{M}$ be a smooth Riemannian manifold of constant sectional curvature. A rank- $k$ generalized immersion of $M$ in $\bar{M}$ is $(n-k)$-developable. Conversely, if $\gamma: M \rightarrow \mathrm{Gr}_{n}(T \bar{M})$ is an $(n-k)$-developable generalized immersion, then at each point $m \in M$, the generalized second fundamental form $\mathbf{I}_{m}$ has rank at most $k$.

Proof. The argument is divided into three steps. 
Step 1: ker II is an involutive distribution. First, the rank assumption implies that ker II is a distribution of rank $n-k$. Next, let $\nabla^{M}$ be any torsion-free connection on $M$. Define

$$
\nabla \mathrm{II}: \mathfrak{X}(M) \times \mathfrak{X}(M) \times \Gamma(\mathrm{GT}(M)) \rightarrow \Gamma(\mathrm{GN}(M))
$$

by the formula

$$
\nabla \mathrm{II}(X, Y, \sigma)=\nabla_{X}^{\perp} \mathrm{II}(Y, \sigma)-\mathrm{II}\left(\nabla_{X}^{M} Y, \sigma\right)-\mathrm{II}\left(Y, \nabla_{X} \sigma\right) .
$$

Lemma 3.1. If $\nabla$ II is symmetric, then $[X, Y] \in \Gamma$ (ker II) for all $X, Y \in \Gamma($ ker II). Proof. When $Y \in \Gamma($ ker II), (3-1) reduces to

$$
\nabla \Pi(X, Y, \sigma)=-\Pi\left(\nabla_{X}^{M} Y, \sigma\right) .
$$

Similarly, when $X \in \Gamma($ ker II $)$

$$
\nabla \mathrm{II}(Y, X, \sigma)=-\mathrm{II}\left(\nabla_{Y}^{M} X, \sigma\right) .
$$

So, by the symmetry of $\nabla$ II and the fact that $\nabla^{M}$ is torsion-free, it follows that

$$
0=\nabla \Pi(X, Y, \sigma)-\nabla \Pi(Y, X, \sigma)=-\Pi\left(\nabla_{X}^{M} Y-\nabla_{Y}^{M} X, \sigma\right)=-\Pi([X, Y], \sigma) .
$$

Thus $[X, Y] \in \Gamma($ ker II $)$.

The remainder of step 1 is implied by the following two lemmas.

Lemma 3.2. The tensor $\nabla$ II is symmetric if and only if the generalized tangent bundle $\mathrm{GT}(M)$ is an invariant subbundle of the curvature tensor.

Lemma 3.3. If $\bar{M}$ has constant sectional curvature, then $\mathrm{GT}(M)$ is an invariant subbundle of the curvature tensor.

Proof of Lemma 3.2. Let $\langle\cdot, \cdot\rangle$ denote the pullback of the Riemannian metric on $\bar{M}$ to the bundle $\mathbb{B}=\left(\gamma^{0}\right)^{*}(T \bar{M})$. To show that $\nabla \mathrm{II}$ is symmetric, it suffices to prove that

$$
\langle\nabla \mathrm{II}(X, Y, \sigma)-\nabla \mathrm{II}(Y, X, \sigma), \eta\rangle=0
$$

for all $X, Y \in \mathfrak{X}(M), \sigma \in \Gamma(\mathrm{GT}(M))$, and $\eta \in \Gamma(\mathrm{GN}(M))$. Let $\bar{R}$ denote the curvature of the connection $\bar{\nabla}$ in $\mathbb{B}$. We first show that the skew-symmetric part of $\nabla \mathrm{II}(X, Y, \cdot)$ is the $\mathrm{GT}(M)$-component of $\bar{R}(X, Y)$ restricted to $\mathrm{GT}(M)$, that is,

$$
\langle\nabla \mathrm{II}(X, Y, \sigma)-\nabla \mathrm{II}(Y, X, \sigma), \eta\rangle=\langle\bar{R}(X, Y) \sigma, \eta\rangle .
$$

To begin,

$$
\begin{aligned}
& \langle\nabla \mathrm{II}(X, Y, \sigma)-\nabla \mathrm{II}(Y, X, \sigma), \eta\rangle=\left\langle\nabla_{X}^{\perp} \mathrm{II}(Y, \sigma)-\nabla_{Y}^{\perp} \mathrm{II}(X, \sigma), \eta\right\rangle \\
& \quad-\left\langle\mathrm{II}\left(\nabla_{X}^{M} Y, \sigma\right)-\mathrm{II}\left(\nabla_{Y}^{M} X, \sigma\right), \eta\right\rangle-\left\langle\mathrm{II}\left(Y, \nabla_{X} \sigma\right)-\mathrm{II}\left(X, \nabla_{Y} \sigma\right), \eta\right\rangle .
\end{aligned}
$$


The three summands on the right side of (3-3) can be simplified as follows: By applying the definition of $\nabla^{\perp}$,

$$
\nabla_{X}^{\perp} \mathrm{II}(Y, \sigma)=\bar{\nabla}_{X} \mathrm{II}(Y, \sigma)-\Pi^{\perp}(X, \Pi(Y, \sigma)) .
$$

Because $\mathrm{II}^{\perp}(X, \mathrm{II}(Y, \sigma)) \in \Gamma(\mathrm{GT}(M))$ and $\mathrm{GT}(M) \perp \mathrm{GN}(M)$, we see that

$$
\left\langle\nabla_{X}^{\perp} \Pi(Y, \sigma), \eta\right\rangle=\left\langle\bar{\nabla}_{X} \Pi(Y, \sigma), \eta\right\rangle .
$$

Thus, the first summand on the right side of (3-3) is given as

$$
\left\langle\nabla_{X}^{\perp} \Pi(Y, \sigma)-\nabla_{Y}^{\perp} \Pi(X, \sigma), \eta\right\rangle=\left\langle\bar{\nabla}_{X} \Pi(Y, \sigma)-\bar{\nabla}_{Y} \Pi(X, \sigma), \eta\right\rangle .
$$

Next, since $\nabla^{M}$ is torsion-free, the second summand on the right side of (3-3) becomes

$$
\left\langle\mathrm{II}\left(\nabla_{X}^{M} Y-\nabla_{Y}^{M} X, \sigma\right), \eta\right\rangle=\langle\mathrm{II}([X, Y], \sigma), \eta\rangle .
$$

For the third summand of (3-3), it follows both from the definition of II and from $\mathrm{GT}(M) \perp \mathrm{GN}(M)$ that

$$
\left\langle\mathrm{II}\left(Y, \nabla_{X} \sigma\right)-\mathrm{II}\left(X, \nabla_{Y} \sigma\right), \eta\right\rangle=\left\langle\bar{\nabla}_{Y} \nabla_{X} \sigma-\bar{\nabla}_{X} \nabla_{Y} \sigma, \eta\right\rangle .
$$

Rewriting (3-3), we get

$$
\begin{array}{r}
\langle\nabla \mathrm{II}(X, Y, \sigma)-\nabla \mathrm{II}(Y, X, \sigma), \eta\rangle=\left\langle\bar{\nabla}_{X} \mathrm{II}(Y, \sigma)-\bar{\nabla}_{Y} \mathrm{II}(X, \sigma), \eta\right\rangle \\
-\langle\mathrm{II}([X, Y], \sigma), \eta\rangle-\left\langle\bar{\nabla}_{Y} \nabla_{X} \sigma-\bar{\nabla}_{X} \nabla_{Y} \sigma, \eta\right\rangle .
\end{array}
$$

Next, by the definition of II,

$$
\begin{aligned}
& \bar{\nabla}_{X} \mathrm{I}(Y, \sigma)-\bar{\nabla}_{Y} \mathrm{II}(X, \sigma)-\mathrm{II}([X, Y], \sigma) \\
& \quad=\bar{\nabla}_{X} \bar{\nabla}_{Y} \sigma-\bar{\nabla}_{X} \nabla_{Y} \sigma-\bar{\nabla}_{Y} \bar{\nabla}_{X} \sigma+\bar{\nabla}_{Y} \nabla_{X} \sigma-\bar{\nabla}_{[X, Y]} \sigma+\nabla_{[X, Y]} \sigma .
\end{aligned}
$$

Plugging this into (3-4) and simplifying in the obvious way gives

$$
\begin{aligned}
\langle\nabla \mathrm{II}(X, Y, \sigma)-\nabla \mathrm{II}(Y, X, \sigma), \eta\rangle & =\left\langle\left(\bar{\nabla}_{X} \bar{\nabla}_{Y}-\bar{\nabla}_{Y} \bar{\nabla}_{X}-\bar{\nabla}_{[X, Y]}\right) \sigma, \eta\right\rangle \\
& =\langle\bar{R}(X, Y) \sigma, \eta\rangle .
\end{aligned}
$$

If $\nabla$ II is symmetric, then (3-2) implies that $\langle\bar{R}(X, Y) \sigma, \eta\rangle=0$. Thus

$$
\bar{R}(X, Y) \sigma \in \Gamma(\mathrm{GT}(M)),
$$

i.e., $\mathrm{GT}(M)$ is an invariant subbundle of $\bar{R}$. Conversely, if $\mathrm{GT}(M)$ is an invariant subbundle of $\bar{R}$, then the right hand side of (3-2) vanishes, so

$$
\langle\mathrm{II}(X, Y, \sigma)-\mathrm{II}(Y, X, \sigma), \eta\rangle=0 .
$$

Hence $\mathrm{II}(X, Y, \sigma)=\mathrm{II}(Y, X, \sigma)$. 
Proof of Lemma 3.3. We prove that if $\bar{M}$ has constant sectional curvature, then $\bar{R}(X, Y) \sigma \in \mathrm{GT}(M)_{m}$ for any $\sigma \in \mathrm{GT}(M)_{m}$ and any pair of tangent vectors $X, Y \in T_{m} M$. Note that $\bar{R}$ is the pullback via $\gamma^{0}$ of the curvature of the LeviCivita connection on $\bar{M}$; the same symbol $\bar{R}$ is used for both curvatures. Thus if $\sigma=(m, s) \in\left(\gamma^{0}\right)^{*}(T \bar{M})$ then

$$
\bar{R}_{m}(X, Y) \sigma=\bar{R}_{\gamma^{0}(m)}\left(T \gamma^{0}(X), T \gamma^{0}(Y)\right) s .
$$

Because $\bar{M}$ has constant sectional curvature, there is a constant $\lambda$ such that for any $x, y, z \in T_{\gamma^{0}(m)} \bar{M}$,

$$
\bar{R}_{\gamma^{0}(m)}(x, y) z=\lambda(\langle z, x\rangle y-\langle z, y\rangle x) ;
$$

see [Kobayashi and Nomizu 1963, (5.2.3)]. Next, by the definition of a generalized immersion, $\gamma$ is annihilated by contact; so $T \gamma^{0}\left(T_{m} M\right) \subset \gamma(m)=\mathrm{GT}(M)_{m}$. It follows that

$$
\bar{R}_{m}(X, Y) \sigma=\lambda\left(\left\langle s, T \gamma^{0}(X)\right\rangle T \gamma^{0}(Y)-\left\langle s, T \gamma^{0}(Y)\right\rangle T \gamma^{0}(X)\right) \in \mathrm{GT}(M)_{m} .
$$

In other words, $\mathrm{GT}(M)_{m}$ is an invariant subspace of $\bar{R}_{m}(X, Y)$.

By the Frobenius Theorem and the involutivity of ker II, we get a foliation $\left\{\mathscr{L}_{\alpha}\right\}$ of $M$ where the leaves are $(n-k)$-dimensional and where $T_{m}\left(\mathscr{L}_{\alpha}\right)=\operatorname{ker} \mathrm{I}_{m}$. We call this the (ker II)-foliation.

Step 2: The images of the leaves $\mathscr{L}_{\boldsymbol{\alpha}}$ under $\boldsymbol{\gamma}$ are horizontal. Let $m(t)$ be an integral curve of the distribution ker II. Let $\bar{m}(t)=\left(\gamma^{0} \circ m\right)(t)$ and denote parallel translation by $\bar{\tau}_{t}: T_{\bar{m}(0)} \bar{M} \rightarrow T_{\bar{m}(t)} \bar{M}$. By (2-5), it suffices to show that the image curve $P(t)=(\gamma \circ m)(t)$ has the form

$$
P(t)=\bar{\tau}_{t}(P(0)) .
$$

In other words, $P(t)$ is the parallel translate of the initial $n$-plane $P(0)$ along $\bar{m}(t)$.

Let $\bar{\tau}_{t}$ also denote the parallel translation in $\mathbb{B}$ along $m(t)$ and let $\tau_{t}$ denote the parallel translation in GT $(M)$ along $m(t)$ corresponding to the induced connection $\nabla$. Since $\mathrm{II}\left(m^{\prime}(t), \cdot\right)=0$, the map $\tau_{t}: \mathrm{GT}(M)_{m(0)} \rightarrow \mathrm{GT}(M)_{m(t)}$ is just the restriction of the linear map $\bar{\tau}_{t}: \mathbb{B}_{m(0)} \rightarrow \mathbb{B}_{m(t)}$ to $\mathrm{GT}(M)_{m(0)}$.

Next, recall GT $(M)_{m}=\gamma(m)$ so that

$$
P(t)=\gamma(m(t))=\mathrm{GT}(M)_{m(t)} .
$$

Thus by the observation just above, we conclude that

$$
\bar{\tau}_{t}^{-1}(P(t))=\tau_{t}^{-1}\left(\mathrm{GT}(M)_{m(t)}\right)=\mathrm{GT}(M)_{m(0)}=P(0),
$$

so $P(t)=\bar{\tau}_{t}(P(0))$. It now follows that the image of each leaf $\mathscr{L}_{\alpha}$ under $\gamma$ is horizontal in $\mathrm{Gr}_{n}(T \bar{M})$. 
Step 3: Converse. Suppose the generalized immersion is $(n-k)$-developable. Let $\mathscr{L}_{\alpha}$ denote a typical leaf of the foliation and let $h \in T_{m} \mathscr{L}_{\alpha}$. Since $T \gamma(h)$ is a horizontal tangent vector to $\mathrm{Gr}_{n}(T \bar{M})$, Lemma 2.2 gives

$$
\mathbf{I}_{m}(h, \sigma)=(\psi_{\gamma(m)} \underbrace{\theta(T \gamma(h))}_{0}) \cdot \sigma=0 .
$$

Thus, $T_{m} \mathscr{L}_{\alpha} \subset \operatorname{ker} \mathrm{II}_{m}$ and so the dimension of $\operatorname{ker} \mathrm{II}_{m}$ is at least $n-k$. Equivalently, the rank of $I_{m}$ is at most $k$.

\section{The total geodesy question}

In the previous section we described the natural foliation that occurs when a generalized immersion has less than full rank. In this section we determine sufficient conditions for the leaves of the foliation to be totally geodesic. Example 6.3 shows that these conditions are reasonably sharp.

Theorem 2. Let $\bar{M}$ be a smooth Riemannian manifold of constant sectional curvature and let $\gamma: M \rightarrow \operatorname{Gr}_{n}(T \bar{M})$ be a rank $k$ generalized immersion. Then the immersed leaves $\overline{\mathscr{L}}_{\alpha}$ of the (ker II)-foliation are totally geodesic in each of the following cases:

(1) $M$ is any classically immersed submanifold of $\bar{M}$;

(2) $M$ is any codimension-1 generalized immersion;

(3) ker II has codimension 1, i.e., rank II $=1$.

The proof of Theorem 2 requires several preliminary lemmas.

Lemma 4.1. The tangent map of $\gamma^{0}=\pi \circ \gamma$ induces a homomorphism of vector bundles over the identity,

$$
\widetilde{T \gamma^{0}}: T M \rightarrow \mathbb{B}=\left(\gamma^{0}\right)^{*}(T \bar{M}) .
$$

The image of $\widetilde{T \gamma^{0}}$ lies in $\mathrm{GT}(M)$. Notationally, if $h \in T M$ then $\tilde{h}=\widetilde{T \gamma^{0}}(h)$.

Proof. Let $\pi_{M}: T M \rightarrow M$ denote the tangent bundle projection. Then

$$
\tilde{h}=\left(\pi_{M}(h), T \gamma^{0} \cdot h\right) .
$$

By assumption, the immersion $\gamma: M \rightarrow \mathrm{Gr}_{n}(T \bar{M})$ is annihilated by contact. So

$$
T \gamma^{0} \cdot h=T \pi \cdot T \gamma \cdot h \in \gamma(m) \text { for } m \in M \text { and } h \in T_{m} M .
$$

On the other hand, by (2-2), GT $(M)_{m}=\mathbb{U}_{\gamma(m)}=\gamma(m)$.

It follows from the lemma that $\widetilde{T \gamma^{0}}$ induces a map on sections, namely,

$$
X \mapsto \tilde{X}=\widetilde{T \gamma^{0}}(X)
$$

where $\tilde{X} \in \Gamma(\mathrm{GT}(M))$ is interpreted pointwise. 
Given any connection $\bar{\nabla}$ on $\bar{M}$, let $\bar{T}$ denote its torsion tensor. Then the pullback of $\bar{T}$ to $M$ by $\gamma^{0}$ is the $\mathbb{B}$-valued 2-form given by

$$
T(X, Y)=\bar{\nabla}_{X} \tilde{Y}-\bar{\nabla}_{Y} \tilde{X}-\widehat{[X, Y]}
$$

for all vector fields $X, Y \in \mathfrak{X}(M)$. As before, $\bar{\nabla}$ denotes both the connection in $T \bar{M}$ and in the pullback bundle $\mathbb{B}$.

Assume that $\bar{\nabla}$ is torsion free, i.e., $\bar{T}=0$. Then

$$
\bar{\nabla}_{X} \tilde{Y}-\bar{\nabla}_{Y} \tilde{X}=\widetilde{[X, Y]}
$$

for all vector fields $X, Y \in \mathfrak{X}(M)$. As a consequence:

Lemma 4.2. The generalized second fundamental form satisfies the property:

$$
\mathrm{II}(X, \tilde{Y})=\mathrm{I}(Y, \tilde{X}) \quad \text { for all } X, Y \in \mathfrak{X}(M) .
$$

Proof. Combining the definition of II with (4-1),

$$
\mathrm{II}(X, \tilde{Y})=\operatorname{nor}\left(\bar{\nabla}_{X} \tilde{Y}\right)=\operatorname{nor}\left(\bar{\nabla}_{Y} \tilde{X}+\widetilde{[X, Y]}\right)=\mathrm{II}(Y, \tilde{X})+\operatorname{nor} \widetilde{[X, Y]} .
$$

By Lemma 4.1, $\widetilde{[X, Y]} \in \Gamma(\mathrm{GT}(M))$. Thus nor $\widetilde{[X, Y]}=0$.

Lemma 4.3. The restriction of $\widetilde{T \gamma^{0}}$ to ker II defines an injective bundle morphism

$$
\widetilde{T \gamma^{0}}: \operatorname{ker} \text { II } \rightarrow \mathrm{GT}(M)
$$

whose image lies in $R$ ker II.

Proof. Let $h \in \operatorname{ker} \mathrm{II}_{m}$. Then by Lemma 4.2,

$$
\mathrm{II}_{m}(v, \tilde{h})=\Pi_{m}(h, \tilde{v})=0
$$

for all $v \in T_{m} M$. So by definition, $\tilde{h} \in R$ ker $\mathrm{II}_{m}$. On the other hand, suppose that $h \in \operatorname{ker} \mathbb{I}_{m}$ and $\tilde{h}=0$. Then $T \gamma^{0}(h)=0$ and thus $T \gamma(h)$ is a vertical tangent vector. However, $T \gamma(h)$ is also a horizontal tangent vector by Theorem 1. Consequently, $T \gamma(h)=0$, and thus $h=0$ because $T \gamma$ is injective.

Lemma 4.4. For each $X, Y \in \Gamma($ ker II) and for all $Z \in \mathfrak{X}(M)$,

$$
\mathrm{II}\left(Z, \bar{\nabla}_{X} \tilde{Y}\right)=0 .
$$

Proof. By the definition of $\nabla$ II (see (3-1))

$$
\nabla \Pi(X, Z, \tilde{Y})=\nabla_{X}^{\perp} \Pi(Z, \tilde{Y})-\Pi\left(\nabla_{X}^{M} Z, \tilde{Y}\right)-\Pi\left(Z, \nabla_{X} \tilde{Y}\right) .
$$

Combining Lemma 4.3 with the assumption that $Y \in \Gamma($ ker II $)$,

$$
\mathrm{II}(Z, \tilde{Y})=0 \quad \text { and } \quad \operatorname{II}\left(\nabla_{X}^{M} Z, \tilde{Y}\right)=0 .
$$


Thus,

$$
\nabla \mathrm{II}(X, Z, \tilde{Y})=-\mathrm{II}\left(Z, \bar{\nabla}_{X} \tilde{Y}\right) .
$$

On the other hand, Lemmas 3.2 and 3.3 show that $\nabla$ II is symmetric when $\bar{M}$ has constant sectional curvature. Thus $\nabla \mathrm{II}(X, Z, \tilde{Y})=\nabla \mathrm{II}(Z, X, \tilde{Y})$. However, in the expansion of $\nabla \Pi(Z, X, \tilde{Y})$, the terms $\mathrm{II}(X, \tilde{Y}), \Pi\left(\nabla_{Z}^{M} X, \tilde{Y}\right)$, and $\mathrm{II}\left(X, \bar{\nabla}_{Z} \tilde{Y}\right)$ all vanish. It follows that $\nabla \mathrm{II}(Z, X, \tilde{Y})=0$ and thus $\operatorname{II}\left(Z, \bar{\nabla}_{X} \tilde{Y}\right)=0$.

Let $\left(\operatorname{ker} \mathbf{I}_{m}\right)^{\sim}$ denote the image of ker $\mathbf{I}_{m}$ in $R$ ker $\mathbf{I}_{m}$.

Lemma 4.5. If $(\text { ker II })^{\sim}=R$ ker II, then the immersed leaves $\overline{\mathscr{L}}_{\alpha}$ are totally geodesic submanifolds of $\bar{M}$.

Proof. Denote by $\nabla^{\alpha}$ the connection on $\overline{\mathscr{L}}_{\alpha}$ induced from $\bar{\nabla}$ and by II $^{\alpha}$ its second fundamental form. Then for all $W, Z \in \mathfrak{X}\left(\overline{\mathscr{L}}_{\alpha}\right)$,

$$
\bar{\nabla}_{W} Z=\nabla_{W}^{\alpha} Z+\Pi^{\alpha}(W, Z) .
$$

By definition, $\overline{\mathscr{L}}_{\alpha}$ is totally geodesic in $\bar{M}$ if and only if II $^{\alpha}=0$, or equivalently, if and only if

$$
\bar{\nabla}_{W} Z=\nabla_{W}^{\alpha} Z
$$

see [O'Neill 1983, (4.12)]. Whether or not $\overline{\mathscr{L}}_{\alpha}$ is totally geodesic is a local question. So since the leaf $\mathscr{L}_{\alpha}$ immerses, we may assume it is embedded. Thus, each vector field of $\overline{\mathscr{L}}_{\alpha}$ is $\gamma^{0}$-related from a unique vector field of $\mathscr{L}_{\alpha}$. Given $W, Z \in \mathfrak{X}\left(\overline{\mathscr{L}}_{\alpha}\right)$, let $X, Y \in \mathfrak{X}\left(\mathscr{L}_{\alpha}\right)$ be the unique vector fields such that for each $m \in \mathscr{L}_{\alpha}$, we have $T \gamma^{0} \cdot X(m)=W\left(\gamma^{0}(m)\right)$ and $T \gamma^{0} \cdot Y(m)=Z\left(\gamma^{0}(m)\right)$. By Theorem 1, vector fields of $\mathscr{L}_{\alpha}$ can be extended to sections of ker II; hence it is assumed at the outset that $X, Y \in \Gamma(\operatorname{ker}$ II $)$.

Along $\mathscr{L}_{\alpha}, \bar{\nabla}_{X} \tilde{Y}$ and $\bar{\nabla}_{W} Z$ agree in the following sense: let $m \in \mathscr{L}_{\alpha}$ and let $m(t)$ be an integral curve of $X$ with origin $m$. Next, let $\bar{\tau}_{t}: \mathbb{B}_{m} \rightarrow \mathbb{B}_{m(t)}$ denote parallel translation in $\mathbb{B}$ along $m(t)$. Using the same symbol,

$$
\bar{\tau}_{t}: T_{\gamma^{0}(m)} \bar{M} \rightarrow T_{\gamma^{0}(m(t))} \bar{M}
$$

also denotes parallel translation in $T \bar{M}$ along the curve $\left(\gamma^{0} \circ m\right)(t)$. Then $\bar{\nabla}_{X} \tilde{Y}(m)$ is represented by the curve $\bar{\tau}_{t}^{-1} \tilde{Y}(m(t))$. In contrast, $\bar{\nabla}_{W} Z\left(\gamma^{0}(m)\right)$ is represented by the curve $\bar{\tau}_{t}^{-1} Z\left(\gamma^{0}(m(t))\right)$, because $\left(\gamma^{0} \circ m\right)(t)$ is an integral curve of $Z$ through $m$. In particular,

$$
\begin{aligned}
\bar{\tau}_{t}^{-1} \tilde{Y}(m(t))=\bar{\tau}_{t}^{-1}\left(m(t), T \gamma^{0} \cdot Y(m(t))\right) & =\bar{\tau}_{t}^{-1}\left(m(t), Z\left(\gamma^{0}(m(t))\right)\right) \\
& =\left(\gamma(m), \bar{\tau}_{t}^{-1} Z\left(\gamma^{0}(m(t))\right)\right),
\end{aligned}
$$

so that

$$
\bar{\nabla}_{X} \tilde{Y}(m)=\left(m, \bar{\nabla}_{W} Z(m)\right)
$$


for each $m \in \mathscr{L}_{\alpha}$.

By Lemma 4.4, $\bar{\nabla}_{X} \tilde{Y}(m) \in R$ ker $\mathbb{I}_{m}$ for each $m \in M$. So by hypothesis, there is a unique vector field $U \in \Gamma$ (ker II) such that $\tilde{U}=\bar{\nabla}_{X} \tilde{Y}$. But $U$ restricts to a vector field along $\mathscr{L}_{\alpha}$, i.e., $U(m) \in \operatorname{ker} \mathbb{I}_{m}=T_{m} \mathscr{L}_{\alpha}$. Hence $T \gamma^{0} \cdot U(m) \in T \gamma^{0}\left(T_{m} \mathscr{L}_{\alpha}\right)=$ $T_{\gamma^{0}(m)} \overline{\mathscr{L}}_{\alpha}$. Equation (4-2) then implies

$$
\bar{\nabla}_{W} Z(m)=T \gamma^{0} \cdot U(m) \in T_{\gamma^{0}(m)} \overline{\mathscr{L}}_{\alpha} .
$$

Thus $\bar{\nabla}_{W} Z(m)=\bar{\nabla}_{W}^{\alpha} Z(m)$.

Proof of Theorem 2. We will show that, for each of the three cases given in the statement of the theorem, the hypothesis of Lemma 4.5 is valid.

Case 1. Let $M$ be a classically immersed submanifold of $\bar{M}$. Then $\gamma$ is the prolongation of the immersion $\gamma^{0}: M \rightarrow \bar{M}$, that is, for each $m \in M, \gamma(m)=T \gamma^{0}\left(T_{m} M\right)$. Since $\mathrm{GT}(M)_{m}=\gamma(m)$, for each $\sigma \in \mathrm{GT}(M)_{m}$, there is a unique $v \in T_{m} M$ such that

$$
\sigma=\left(m, T \gamma^{0}(v)\right)=\tilde{v} .
$$

By Lemma 4.2, for each $h \in T_{m} M$,

$$
\mathbb{I}_{m}(h, \sigma)=\mathbb{I}_{m}(h, \tilde{v})=\Pi_{m}(v, \tilde{h}) .
$$

So if $\sigma=\tilde{v} \in R$ ker $\mathrm{I}_{m}$, we conclude from the observations above that $\mathrm{I}_{m}(v, \tilde{h})=0$ for all $\tilde{h} \in \widetilde{T \gamma^{0}}\left(T_{m} M\right)=\mathrm{GT}(M)_{m}$. Thus $\left(\operatorname{ker} \mathrm{I}_{m}\right)^{\sim}=R$ ker $\mathrm{II}_{m}$.

Case 2. Given $m \in M$, the constant rank $k$ assumption on the generalized immersion implies $h \mapsto \mathbb{I}_{m}(h, \cdot)$ is a linear map

$$
T_{m} M \rightarrow L\left(\mathrm{GT}(M)_{m}, \mathrm{GN}(M)_{m}\right)
$$

whose image has dimension $k$. Next, $\operatorname{dim} \operatorname{GN}(M)_{m}=1$ for all $m \in M$ by the codimension-1 hypothesis of case 2 . The remainder of the argument is pointwise.

Given $m \in M$, identify $L\left(\mathrm{GT}(M)_{m}, \mathrm{GN}(M)_{m}\right)$ with $\mathrm{GT}(M)_{m}^{*}$ by choosing a linear isomorphism from $\mathrm{GN}(M)_{m}$ to $\mathbb{R}$. By Grassmann duality (the obvious generalization of projective duality [Arnol'd 1988]), the subspace

$$
\left\{\mathrm{II}_{m}(h, \cdot) \mid h \in T_{m} M\right\} \subset \mathrm{GT}(M)_{m}^{*}
$$

is dual to

$$
R \text { ker } \mathrm{II}_{m}=\cap_{h \in T_{m} M} \operatorname{ker} \mathrm{II}_{m}(h, \cdot) \subset \mathrm{GT}(M)_{m} .
$$

As the former space has dimension $k$, the latter has dimension $n-k$. On the other hand, in Lemma 4.3 we showed that $\widetilde{T \gamma^{0}}$ maps ker II $_{m}$ injectively to $R$ ker $\mathbb{I}_{m}$. So since $\operatorname{ker} \mathbf{I}_{m}$ has dimension $n-k$, its image $\left(\operatorname{ker} \mathrm{I}_{m}\right)^{\sim}$ in $R$ ker $\mathbf{I}_{m}$ is all of $R$ ker $\mathbb{I}_{m}$. 
Case 3. Suppose ker $I_{m}$ has codimension 1 . Then (ker $\left.I_{m}\right)^{\sim}$ is an $(n-1)$-dimensional subspace of both $R$ ker $\mathrm{II}_{m}$ and GT $(M)_{m}$. Because $\mathrm{GT}(M)_{m}$ has dimension $n$, either $\left(\operatorname{ker} \mathrm{I}_{m}\right)^{\sim}=R$ ker $\mathrm{I}_{m}$ or $R$ ker $\mathrm{I}_{m}=\mathrm{GT}(M)_{m}$. Suppose the latter is true. Then $\mathbb{I}_{m}(h, \sigma)=0$ for $\sigma \in \mathrm{GT}(M)_{m}$ and $h \in T_{m} M$. Thus, $T_{m} M=\operatorname{ker} \mathbb{I}_{m}$ which contradicts the assumption $\operatorname{dim}\left(\operatorname{ker} \mathrm{II}_{m}\right)=n-1$.

The proof of Theorem 2 is now complete.

Note that the case where rank $I I=0$ is also covered by Theorem 2. If rank $I I=0$, then $\operatorname{ker} \mathrm{II}_{m}=T_{m} M$, so all of $M$ is a single leaf. Since the leaves immerse in $\bar{M}$, we are in the classical situation.

\section{More on jets}

The goal of this section is to prove that the (ker II)-foliation has local cross-sections in the case where $\bar{M}$ is a finite dimensional real vector space $E$ of dimension $n+\ell$ with the canonical flat connection (see Theorem 3). This will guarantee that the leaf space $M / \mathscr{L}$ is at least a $T_{1}$-manifold, i.e., locally Euclidean but not necessarily Hausdorff. En route to Theorem 3, we develop some additional machinery for vector space computations.

The canonical flat connection $C: T E \oplus T E \rightarrow T(T E)$ is defined by

$$
C(m ; h, x)=(m, x ; h, 0) .
$$

As usual, we use the canonical trivializations $T E=E \times E, T(T E)=T E \times T E$, and so on. The connection $C$ is the Levi-Civita connection for any constant Riemannian metric on $E$, say $\langle\cdot, \cdot\rangle$. The corresponding parallel translations are all trivial: $\bar{\tau}\left(\bar{m}_{1}, x\right)=\left(\bar{m}_{2}, x\right)$ along any curve from $\bar{m}_{1}$ to $\bar{m}_{2}$.

Consider a generalized immersion $\gamma: M \rightarrow \mathrm{Gr}_{n}(T E)$. Using the canonical trivialization for $\mathrm{Gr}_{n}(T E)$, we write

$$
\gamma(m)=\left(\gamma^{0}(m), \mathbf{P}(m)\right) \in \mathrm{Gr}_{n}(T E)=E \times \mathrm{Gr}_{n}(E) .
$$

We call the smooth map $m \mapsto \mathbf{P}(m)$ the Gauss map of the generalized immersion.

The choice of an orthogonal splitting $E=H \oplus K$ with $\operatorname{dim} H=n$ and $\operatorname{dim} K=\ell$ gives rise to a natural chart

$$
\beta: J^{1}(H, K) \rightarrow U \subset \mathrm{Gr}_{n}(T E)=E \times \mathrm{Gr}_{n}(E)
$$

defined by

$$
\beta(x, z, A)=(x+z, \operatorname{graph}(A)),
$$

where $\operatorname{graph}(A)=\{h+A \cdot h \mid h \in H\}$. Here, we use the canonical description of 1 -jets, namely $J^{1}(H, K)=H \times K \times L(H, K)$.

The image $U$ of the mapping $\beta$ is an open dense subset of $\operatorname{Gr}_{n}(T E)$ and, by varying the choice of splitting, we can cover all of $\mathrm{Gr}_{n}(T E)$ by such charts. The set 
$U$ consists of those $n$-planes that are graphical relative to the splitting $E=H \oplus K$. Since any $n$-plane is graphical relative to some splitting, the local behavior of a generalized immersion in $E$ can be understood in terms of a jet space $J^{1}(H, K)$. Thus we will assume that $\gamma: M \rightarrow \mathrm{Gr}_{n}(T E)$ is a generalized immersion whose image lies in the set $U$ defined by a fixed splitting $E=H \oplus K$. Elements of $E$ can then be viewed as pairs $(h, k)$ or as sums $h+k$, as convenient. Moreover, for local computations, the manifold $M$ can be assumed to be a real vector space of dimension $n$.

The contact form. As in the previous sections, let $\pi: \mathrm{Gr}_{n}(T E) \rightarrow E$ be the Grassmann bundle of $n$-planes in the tangent bundle to $E$, let $\mathbb{C} \subset \pi^{*}(T E)$ be the universal $n$-plane bundle over $\operatorname{Gr}_{n}(T E)$, let $\mathbb{U}^{\perp}$ be the corresponding normal bundle, and let $\mathbb{Q}$ be the quotient bundle $\mathbb{Q}=\pi^{*}(T E) / \mathbb{U}$. Recall that the contact form $\omega$ is the $\mathbb{Q}$-valued 1-form on $\operatorname{Gr}_{n}(T E)$ that is defined by $\omega_{P}(v)=T \pi(v)+P$ for $v \in T_{P}\left(\mathrm{Gr}_{n}(T E)\right)$.

Next, we compute $\beta^{*} \omega$. The tangent vector $(h, k, B)$ at $(x, z, A) \in J^{1}(H, K)$ is represented by the curve $c(t)=(x, z, A)+t(h, k, B)$. It follows that

$$
\begin{aligned}
\left(\beta^{*} \omega\right) \cdot[c(t)] & =\omega(T \beta \cdot[c(t)])=T \pi \cdot T \beta \cdot[c(t)]+\beta(c(0)) \\
& =[(x, z)+t(h, k)]+\beta(x, z, A)=((x, z),(h, k)+\operatorname{graph}(A)) .
\end{aligned}
$$

Clearly, since $(h, A \cdot h) \in \operatorname{graph}(A)$ it follows that

$$
(h, k)+\operatorname{graph}(A)=(0, k-A \cdot h)+\operatorname{graph}(A) .
$$

Moreover, $k-A \cdot h$ is the unique representative of the coset $(h, k)+\operatorname{graph}(A)$ that lies in $K$. Thus, the contact form on $\mathrm{Gr}_{n}(T E)$ can be reinterpreted as the $K$-valued 1 -form on $J^{1}(H, K)$ defined by

$$
\omega_{(x, z, A)}(h, k, B)=k-A \cdot h .
$$

Vertical vectors. Because the chart $\beta$ is compatible with the bundle projection $\pi$, the vertical vectors at $(x, z, A) \in J^{1}(H, K)$ are all of the form $(0,0, B)$ for $B \in$ $L(H, K)$. The formula for the generalized second fundamental form in Lemma 2.2 requires that we reinterpret a vector tangent to the fiber of $\pi: \operatorname{Gr}_{n}(T E) \rightarrow E$ at $P$ as a linear mapping $P$ to $P^{\perp}$. So let $(x, z, A(t))$ be a curve representing the vertical vector $(0,0, B)$ at $(x, z, A)$ and let $G(t)=\operatorname{graph}(A(t)) \in \operatorname{Gr}_{n}(E)$. The curve $P(t)=((x, z), G(t))$ then represents $T \beta[(x, z, A(t))]$. As with $A(t)$, we will let the initial plane $P(0)$ be denoted by $P$. Adopting the notation of Lemma 2.1, we have:

Lemma 5.1. If $A(0)=A$ and $A^{\prime}(0)=B$ then

$$
\psi_{P}(T \beta[(x, z, A(t))]) \cdot(h, A \cdot h)=\left(\iota-{ }^{t} A\right)\left(I+A \cdot{ }^{t} A\right)^{-1} \cdot B \cdot h .
$$


Proof. Let $\operatorname{Pr}_{t}^{\perp}$ be the orthogonal projection of $E$ onto $G(t)^{\perp}$. A direct computation using Lemma 2.1 and a variant of Figure 1 gives

$$
\begin{aligned}
\psi_{P}([P(t)]) \cdot(h, A \cdot h) & =-\left.\frac{d}{d t}\right|_{t=0} \operatorname{Pr}_{t}^{\perp}(h, A \cdot h) \\
& =-\left.\frac{d}{d t}\right|_{t=0} \operatorname{Pr}_{t}^{\perp}((h, A \cdot h)-(h, A(t) \cdot h)) \\
& =-\left.\frac{d}{d t}\right|_{t=0} \operatorname{Pr}_{t}^{\perp}(0,(A-A(t)) \cdot h) \\
& =-\left.\frac{d}{d t}\right|_{t=0}\left(\iota-{ }^{t} A(t)\right) \cdot\left(I+A(t) \cdot{ }^{t} A(t)\right)-1 \cdot(A-A(t)) \cdot h \\
& =\left(\iota-{ }^{t} A\right)\left(I+A \cdot{ }^{t} A\right)^{-1} \cdot B \cdot h .
\end{aligned}
$$

The generalized second fundamental form. Let

$$
\gamma(m)=(x(m), z(m), A(m)): M \rightarrow J^{1}(H, K)
$$

be a generalized immersion. By (5-1), annihilation by contact is the requirement that

$$
D z(m) \cdot v=A(m) \cdot D x(m) \cdot v
$$

for all $(m, v) \in T M=M \times M$. The generalized tangent space at $m$ is given by

$$
\mathrm{GT}(M)_{m}=\{(h, A(m) \cdot h) \mid h \in H\} .
$$

Next by lemmas 2.2 and 5.1, the generalized second fundamental form is given by

$$
\begin{aligned}
\mathrm{II} & (m ; v,(h, A(m) \cdot h)) \\
& =\psi_{\beta \circ \gamma(m)}\left(\theta \cdot T \beta_{\gamma(m)}(D x(m) v, D z(m) v, D A(m) v)\right) \cdot(h, A(m) h) \\
& =\psi_{\beta \circ \gamma(m)}(T \beta \cdot[(x(m), z(m), A(m)+t \cdot D A(m) v)]) \cdot(h, A(m) h) \\
& =\left(\iota-{ }^{t} A(m)\right) \cdot\left(I+A(m) \cdot{ }^{t} A(m)\right)^{-1} \cdot D A(m) \cdot v \cdot h .
\end{aligned}
$$

In particular, ker $\mathbf{I}_{m}=\{v \in M \mid D A(m) \cdot v=0 \in L(H, K)\}$.

Local cross-sections. Let $M$ be a smooth manifold with a foliation $\left\{\mathscr{L}_{\alpha}\right\}$. We say the foliation admits slices or local cross-sections if and only if for each $m \in M$, there is a local submanifold $\Sigma=\Sigma_{m}$ of $M$ that intersects each leaf at most once and such that $T_{m} M=T_{m} \Sigma \oplus T_{m} \mathscr{L}_{[m]}$, where $\mathscr{L}_{[m]}$ is the leaf containing $m$. The local slices can be used to define a locally Euclidean structure on $M / \mathscr{L}$. If in addition the equivalence relation is closed so that $M / \mathscr{L}$ is Hausdorff, then we call the foliation regular; see [Abraham et al. 1988, (4.4.9)] and examples 6.4 and 6.5.

Theorem 3. The (ker II)-foliation of a constant rank generalized immersion into a vector space admits local cross-sections.

Proof. Let $\gamma: M \rightarrow \mathrm{Gr}_{n}(T E)$ be a rank- $k$ generalized immersion into a vector space $E$ equipped with the canonical flat connection. Keeping to the notation of 
this section,

$$
\gamma(m)=\left(\gamma^{0}(m), \mathbf{P}(m)\right) \in E \times \mathrm{Gr}_{n}(E),
$$

where $m \mapsto \mathbf{P}(m)$ is the Gauss map of the generalized immersion. It is an immediate corollary of Theorem 1 that the Gauss map is constant along the leaves of the (ker II)-foliation. Next, it will be argued that for each $m \in M$,

$$
\operatorname{ker}\left(T \mathbf{P}: T_{m} M \rightarrow T_{\mathbf{P}(m)} \operatorname{Gr}_{n}(E)\right)=\operatorname{ker} \mathrm{II}_{m} .
$$

This is a pointwise statement, so a local argument using the vector space methods established earlier in the section is appropriate. Let

$$
\gamma(m)=(x(m), z(m), A(m)): M \rightarrow J^{1}(H, K)
$$

be our generalized immersion. Then the Gauss map $\mathbf{P}$ is realized as the smooth function $A: M \rightarrow L(H, K)$ and the principal part of the tangent mapping $T A$ is the derivative $D A: M \rightarrow L(M, L(H, K))$. It is then immediate from (5-2) that ker $\mathrm{II}_{m}=\operatorname{ker} D A(m)$; thus (5-3) follows.

To obtain local slices, let $\varphi: U \rightarrow L \oplus Q$ be a foliation chart centered at a point $m$. We can assume that $\varphi$ is onto so that the connected components $\left(U \cap \mathscr{L}_{\alpha}\right)^{\beta}$ of $U \cap \mathscr{L}_{\alpha}$ are cosets of $L$, that is,

$$
\varphi\left(\left(U \cap \mathscr{L}_{\alpha}\right)^{\beta}\right)=q_{\alpha}^{\beta}+L \text { for appropriate } q_{\alpha}^{\beta} \in Q .
$$

Next, $\widetilde{\Sigma}=\varphi^{-1}(Q)$ is an embedded submanifold of $M$. By consideration of the chart, $T_{m} \widetilde{\Sigma} \cap T_{m} \mathscr{L}_{[m]}=\{0\}$, and so $T_{m} \widetilde{\Sigma} \oplus T_{m} \mathscr{L}_{[m]}=T_{m} M$. It follows that the linear map $T \mathbf{P}: T_{m} \widetilde{\Sigma} \rightarrow T_{\mathbf{P}(m)} \mathrm{Gr}_{n}(E)$ is injective. Otherwise (5-3) would imply that $T_{m} \widetilde{\Sigma} \cap T_{m} \mathscr{L}_{[m]} \neq\{0\}$. Now by the Inverse Function Theorem, there is an open neighborhood $\Sigma_{m}$ of $m$ in $\widetilde{\Sigma}$ such that the map $\mathbf{P}: \Sigma_{m} \rightarrow \operatorname{Gr}_{n}(E)$ is an embedding. Finally, because the Gauss map $\mathbf{P}$ is both constant on leaves and injective on $\Sigma_{m}$, the set $\Sigma_{m}$ intersects each leaf at most once and is thus a local slice.

\section{Examples}

Example 6.1. Let $c(s)$ be a smooth curve in $\mathbb{R}^{3}$, parameterized by arc length whose curvature is everywhere nonzero. Let $(T, N, B)$ be the resulting orthonormal Frenet frame along the curve, that is, $c^{\prime}(s)=T(s), c^{\prime \prime}(s)=T^{\prime}(s)=\kappa(s) N(s)$, and $B(s)=T(s) \times N(s)$. Here, the curvature $\kappa(s)$ is assumed to be strictly positive. Also, the torsion $\tau$ of the curve is defined by $B^{\prime}(s)=-\tau(s) N(s)$.

Consider the tangentially developed surface defined by $c$, i.e., let

$$
\gamma^{0}(s, t)=c(s)+t c^{\prime}(s): \mathbb{R}^{2} \rightarrow \mathbb{R}^{3} .
$$

We have

$$
D \gamma^{0}(s, t) \cdot(h, k)=(h+k) T(s)+t h \kappa(s) N(s),
$$


so $\gamma^{0}(s, t)$ is singular if and only if $t=0$. The nonsingular set is then dense in $\mathbb{R}^{2}$. So by the general principle described in Section 1, there is at most one generalized immersion $\gamma: \mathbb{R}^{2} \rightarrow \mathrm{Gr}_{2}\left(T \mathbb{R}^{3}\right)$ that lifts the mapping $\gamma^{0}$. Indeed by (6-1), the only way to lift $\gamma^{0}$ to a mapping annihilated by contact is to let

$$
\gamma(s, t)=\left(\gamma^{0}(s, t), B(s)^{\perp}\right) \in \mathbb{R}^{3} \times \operatorname{Gr}_{2}\left(\mathbb{R}^{3}\right) .
$$

Recall, $\gamma$ must be the prolongation of $\gamma^{0}$ along the nonsingular set. This mapping is clearly smooth and, by general principles, is annihilated by contact. The only remaining issue is whether $\gamma$ is an immersion. We will prove that $\gamma(s, t)$ is an immersion unless $t=0$ and $\tau(s)=0$. Thus, a generic tangentially developed surface (that is, one where the developing curve has nonvanishing torsion) can be viewed as a generalized immersion in an absolutely canonical way.

The tangent vector $(s, t ; h, k) \in T_{(s, t)} \mathbb{R}^{2}$ is represented by the curve

$$
u \mapsto(s+u h, t+u k),
$$

so that $T \gamma(s, t ; h, k)$ is represented by the curve

$$
u \mapsto\left(\gamma^{0}(s+u h, t+u k), B(s+u h)^{\perp}\right) \in \mathbb{R}^{3} \times \mathrm{Gr}_{2}\left(\mathbb{R}^{3}\right) .
$$

Let $P(u)=B(s+u h)^{\perp}$. By Lemma 2.1, we can interpret the tangent vector to $\mathrm{Gr}_{2}\left(\mathbb{R}^{3}\right)$ represented by the curve $u \mapsto P(u)$ as an element of $L\left(P(0), P(0)^{\perp}\right)$. In this particular case, the projection map $\operatorname{Pr}_{u}^{\perp}$ is given by

$$
\operatorname{Pr}_{u}^{\perp}(v)=\langle v, B(s+u h)\rangle B(s+u h) .
$$

Differentiation at $u=0$ yields

$$
\begin{aligned}
\left.\frac{d}{d u}\right|_{u=0} \operatorname{Pr}_{u}^{\perp}(v) & =\langle v, B(s)\rangle h B^{\prime}(s)+\left\langle v, h B^{\prime}(s)\right\rangle B(s) \\
& =-h \tau(s)\langle v, B(s)\rangle N(s)-h \tau(s)\langle v, N(s)\rangle B(s) .
\end{aligned}
$$

By Lemma 2.1, the tangent vector represented by the curve $u \mapsto P(u)$ corresponds to the linear map

$$
v \mapsto h \tau(s)\langle v, N(s)\rangle B(s)
$$

from $P(0)=B(s)^{\perp}$ to $P(0)^{\perp}=\mathbb{R} \cdot B(s)$. Viewing $T \gamma(s, t ; h, k)$ as an element of $\mathbb{R}^{3} \times L\left(P(0), P(0)^{\perp}\right)$, we have

$$
T \gamma(s, t ; h, k)=\left(D \gamma^{0}(s, t) \cdot(h, k), h \tau(s)\langle\cdot, N(s)\rangle B(s)\right) .
$$

Taking (6-1) into account, the only way that this can be zero without having both $h$ and $k$ equal to zero is if $t=0, \tau(s)=0$, and $h=-k$. 
Example 6.2. Let $c(t)=(x(t), y(t), z(t))$ be a smooth curve in $\mathbb{R}^{3}$, where for convenience, we assume $x^{\prime}(t) \neq 0$ for all $t$. Define a generalized immersion $\gamma: \mathbb{R}^{2} \rightarrow J^{1}\left(\mathbb{R}^{2}, \mathbb{R}\right) \subset \mathrm{Gr}_{2}\left(T \mathbb{R}^{3}\right)$ by

$$
\gamma(s, t)=\left(x(t), y(t), z(t),\left(\frac{z^{\prime}(t)}{x^{\prime}(t)}+s y^{\prime}(t),-s x^{\prime}(t)\right)\right) ;
$$

see Figure 2.

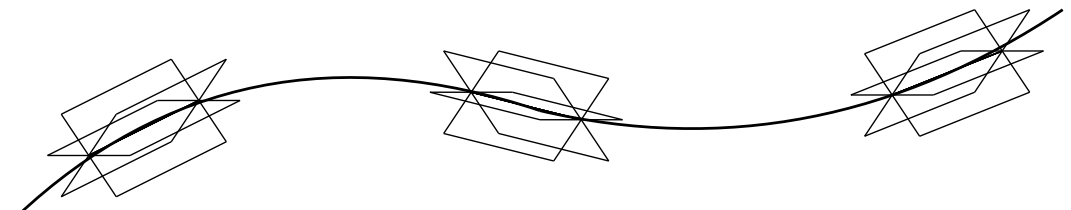

Figure 2. The generalized immersion of Example 6.2.

Let

$$
A(s, t)=\left(\frac{z^{\prime}(t)}{x^{\prime}(t)}+s y^{\prime}(t),-s x^{\prime}(t)\right) .
$$

Computing

$$
D A(s, t)=\left(\left(y^{\prime},-x^{\prime}\right),\left(\frac{x^{\prime} z^{\prime \prime}-z^{\prime} x^{\prime \prime}}{\left(x^{\prime}\right)^{2}}+s y^{\prime \prime},-s x^{\prime \prime}\right)\right)
$$

where for convenience, we omit the explicit dependence on $t$. Since $x^{\prime}(t) \neq 0$ by assumption, we see that $D \gamma(s, t)$ is injective for all $(s, t) \in \mathbb{R}^{2}$, and so $\gamma$ is an immersion. Moreover $\gamma$ is annihilated by contact because, by (5-1),

$$
\omega_{\gamma(s, t)} D \gamma(s, t) \cdot\left(s_{1}, t_{1}\right)=t_{1} z^{\prime}-\left(\frac{z^{\prime}}{x^{\prime}}+s y^{\prime},-s x^{\prime}\right)\left(\begin{array}{l}
t_{1} x^{\prime} \\
t_{1} y^{\prime}
\end{array}\right)=0 .
$$

Next by (5-2), the kernel of the generalized second fundamental form at $(s, t)$ is the same as the kernel of $\widetilde{D A}(s, t)$, where $\widetilde{D A}(s, t)$ is the $2 \times 2$ matrix defined by making the two entries of $D A(s, t)$ into columns. Again since $x^{\prime}(t) \neq 0$, we see that the rank of $D A(s, t)$ is either 1 or 2 , depending on whether the determinant of $\widetilde{D A}(s, t)$ is 0 or not. Computing,

$$
\operatorname{det} \widetilde{D A}(s, t)=s\left(x^{\prime} y^{\prime \prime}-y^{\prime} x^{\prime \prime}\right)+\frac{x^{\prime} z^{\prime \prime}-z^{\prime} x^{\prime \prime}}{x^{\prime}} .
$$

The curvature $\kappa(t)$ of the curve $c$ is zero at $c(t)$ if and only if $c^{\prime}(t)$ and $c^{\prime \prime}(t)$ are linearly dependent, or equivalently, if and only if the cross product $c^{\prime}(t) \times c^{\prime \prime}(t)$ is 0 . Clearly, if $\kappa(t)=0$ then $D A(s, t)$ has rank 1 for all $s$. Conversely by (6-2), 
if $\kappa(t) \neq 0$ and $x^{\prime} y^{\prime \prime}-y^{\prime} x^{\prime \prime} \neq 0$ (not really a restriction if $\kappa(t) \neq 0$ by general position), then $D A(s, t)$ has rank 2 unless

$$
s=\frac{z^{\prime} x^{\prime \prime}-x^{\prime} z^{\prime \prime}}{x^{\prime}\left(x^{\prime} y^{\prime \prime}-y^{\prime} x^{\prime \prime}\right)} .
$$

For this particular value of $s$ we see that

$$
A(s, t)=\left(\frac{z^{\prime} y^{\prime \prime}-y^{\prime} z^{\prime \prime}}{x^{\prime} y^{\prime \prime}-y^{\prime} x^{\prime \prime}}, \frac{x^{\prime} z^{\prime \prime}-z^{\prime} x^{\prime \prime}}{x^{\prime} y^{\prime \prime}-y^{\prime} x^{\prime \prime}}\right) .
$$

The graph of this particular $A(s, t)$ is precisely the osculating plane at $c(t)$.

Example 6.3. Let $J^{1}=J^{1}\left(\mathbb{R}^{3}, \mathbb{R}^{2}\right)$ and let $m=(r, s, t) \in \mathbb{R}^{3}$. Define $\gamma: \mathbb{R}^{3} \rightarrow J^{1}$ by

$$
\gamma(m)=(x(m), z(m), A(m))
$$

where

$$
x(m)=\left(\begin{array}{c}
r \\
r^{2} \\
0
\end{array}\right), \quad z(m)=\left(\begin{array}{l}
0 \\
0
\end{array}\right), \quad \text { and } \quad A(m)=\left(\begin{array}{lll}
0 & 0 & s \\
0 & 0 & t
\end{array}\right) .
$$

We will show that $\gamma$ is a generalized immersion of rank 2. Considering the leaves of the rank- 1 foliation defined by the kernel of the generalized second fundamental form, we will show that the images of the leaves are parabolas in $J^{0}=\mathbb{R}^{3} \times \mathbb{R}^{2}$. Hence they are not totally geodesic submanifolds of $J^{0}$. The example fails the hypotheses of the three cases in Theorem 2. The generalized immersion is not classical; the codimension is 2 ; and the rank of II is 2. Computing,

$$
D \gamma(m) \cdot\left(r_{1}, s_{1}, t_{1}\right)=\left(\left(\begin{array}{c}
r_{1} \\
2 r \\
r
\end{array} r_{1}\right),\left(\begin{array}{l}
0 \\
0
\end{array}\right),\left(\begin{array}{lll}
0 & 0 & s_{1} \\
0 & 0 & t_{1}
\end{array}\right)\right),
$$

so that

$$
\left(r_{1}, s_{1}, t_{1}\right) \in \operatorname{ker}(D \gamma(m)) \quad \Longleftrightarrow \quad\left(r_{1}, s_{1}, t_{1}\right)=(0,0,0) .
$$

Thus, $\gamma$ is a smooth immersion. Next, we show that $\gamma$ is annihilated by contact. According to Section 5, this is verified by the computation

$$
\omega_{\gamma(m)}\left(D \gamma(m) \cdot\left(r_{1}, s_{1}, t_{1}\right)\right)=\left(\begin{array}{l}
0 \\
0
\end{array}\right)-\left(\begin{array}{lll}
0 & 0 & s \\
0 & 0 & t
\end{array}\right)\left(\begin{array}{c}
r_{1} \\
2 r r_{1} \\
0
\end{array}\right)=\left(\begin{array}{l}
0 \\
0
\end{array}\right) .
$$

The generalized second fundamental form of $\gamma$ is given as follows: Let $v=$ $\left(r_{1}, s_{1}, t_{1}\right) \in \mathbb{R}^{3}$. Then by equation (5-2)

$$
\mathrm{II}_{m}: \mathbb{R}^{3} \times \mathrm{GT}_{m} \rightarrow \mathrm{GN}_{m}
$$


is the bilinear map

$$
\mathrm{II}_{m}(v,(h, A(m) \cdot h))=\left(\iota-{ }^{t} A(m)\right) \cdot\left(I+A(m) \cdot{ }^{t} A(m)\right)^{-1} \cdot D A(m) \cdot v \cdot h
$$

and the kernel of II is $\left\{v \in \mathbb{R}^{3} \mid D A(m) \cdot v=0\right\}$. As

$$
D A(m) \cdot v=\left(\begin{array}{lll}
0 & 0 & s_{1} \\
0 & 0 & t_{1}
\end{array}\right)
$$

it follows that

$$
v \in \operatorname{ker} \Pi_{m} \quad \Longleftrightarrow \quad v=\left(r_{1}, 0,0\right) .
$$

Thus ker $\mathbb{I}_{m}$ is 1 -dimensional for all $m \in \mathbb{R}^{3}$. The leaves of the foliation are lines of the form

$$
\mathscr{L}_{(s, t)}=\{(x, s, t) \mid x \in \mathbb{R}\} .
$$

Next,

$$
D \gamma^{0}(m) \cdot\left(r_{1}, 0,0\right)=\left(\left(\begin{array}{c}
r_{1} \\
2 r r_{1} \\
0
\end{array}\right),\left(\begin{array}{l}
0 \\
0
\end{array}\right)\right) \text {. }
$$

Clearly then,

$$
D \gamma^{0}(m) \cdot\left(r_{1}, 0,0\right)=0 \quad \Longleftrightarrow \quad r_{1}=0 .
$$

Thus, $D \gamma^{0}(m)$ is injective on ker $\Pi_{m}$ and the restriction of $\gamma^{0}$ to $\mathscr{L}_{(s, t)}$ is an immersion. Moreover, the immersed leaves

$$
\gamma^{0}\left(\mathscr{L}_{(s, t)}\right)=\left\{\left(\left(\begin{array}{c}
x \\
x^{2} \\
0
\end{array}\right),\left(\begin{array}{l}
0 \\
0
\end{array}\right)\right) \mid x \in \mathbb{R}\right\}
$$

are parabolas, which are not totally geodesic submanifolds of $J^{0}$.

Example 6.4. The torn trough (Figure 3, left) illustrates limits to the regularity of the foliation defined by the (ker II)-distribution. Immerse a plane in $\mathbb{R}^{3}$ as a cylindrical trough. The resulting parallel rulings then form the (ker II)-foliation. However by introducing a tear in the surface, the resulting leaf space (Figure 3, right) ceases to be Hausdorff although the local cross-sections from Theorem 3 guarantee that the resulting leaf space is a locally Euclidean $T_{1}$-manifold. The Euclidean neighborhoods $U_{A}$ and $U_{B}$ of the leaves $A$ and $B$ always intersect.

Example 6.5. Let $\lambda \in \mathbb{C}$ be a complex number of length 1 that corresponds to a rotation by an irrational multiple of $\pi$. Use $\lambda$ to define a properly discontinuous action of $\mathbb{Z}$ on $\mathbb{R} \times \mathbb{C}$ by

$$
n \cdot(t, z)=\left(t+n, \lambda^{n} z\right)
$$



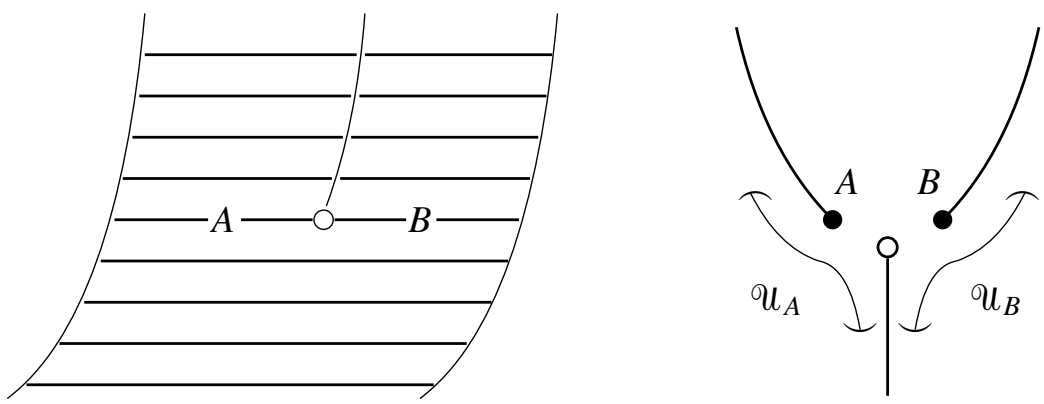

Figure 3. The torn trough and its associated leaf space.

Since the action is properly discontinuous, the quotient

$$
\bar{M}=\mathbb{R} \times \mathbb{C} /(t, z) \sim n \cdot(t, z)
$$

is a manifold. Moreover because the action is by isometries, the quotient inherits the flat Euclidean geometry of $\mathbb{R} \times \mathbb{C}$. Figure 4 represents a rank-1 immersion of the 2-torus into the manifold $\bar{M}$. The figure shows a fundamental domain for the action - the manifold $\bar{M}$ is obtained by identifying points on the left side with points on the right side, after an appropriate rotation. Because the torus inherits the flat geometry of a cylinder, the leaves of the (ker II)-foliation form irrational windings of the torus, as is displayed in the figure. In this case, as with any foliation all of whose leaves are dense, the topology of the leaf space is trivial. In particular, it is not locally Euclidean.

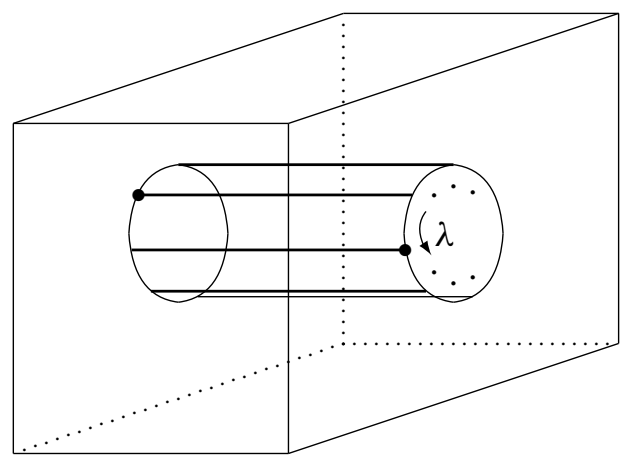

Figure 4. An irrational winding of the torus as a (ker II)-foliation: the space $\bar{M}=[0,1] \times \mathbb{C} / \sim$ where $(0, z) \sim(1, \lambda z)$ for all $z \in \mathbb{C}$. 


\section{References}

[Abraham et al. 1988] R. Abraham, J. E. Marsden, and T. Ratiu, Manifolds, tensor analysis, and applications, Applied Math. Sciences 75, Springer, New York, 1988. MR 89f:58001 Zbl 0875.58002

[Alekseevskij et al. 1991] D. V. Alekseevskij, A. M. Vinogradov, and V. V. Lychagin, Geometry I: basic ideas and concepts of differential geometry, Encyclopaedia of Mathematical Sciences 28, Springer, Berlin, 1991. MR 95i:53001a Zbl 0763.58001

[Arnol'd 1988] V. I. Arnol'd, Geometrical methods in the theory of ordinary differential equations, Grundlehren der Mathematischen Wissenschaften 250, Springer, New York, 1988. MR 89h:58049 Zbl 0507.34003

[Binz et al. 1988] E. Binz, J. Śniatycki, and H. Fischer, Geometry of classical fields, North-Holland Mathematics Studies 154, North-Holland, Amsterdam, 1988. MR 90a:58045 Zbl 0675.53065

[Dieudonné 1972] J. Dieudonné, Treatise on analysis, vol. III, Academic Press, New York, 1972. MR 50 \#3261 Zbl 0268.58001

[Dieudonné 1974] J. Dieudonné, Treatise on analysis, vol. IV, Academic Press, New York, 1974. MR 50 \#3261 Zbl 0292.58001

[Fisher and Laquer 1999] R. J. Fisher and H. T. Laquer, "Second order tangent vectors in Riemannian geometry”, J. Korean Math. Soc. 36:5 (1999), 959-1008. MR 2000g:53014 Zbl 0978.53076

[Jensen and Rigoli 1989] G. R. Jensen and M. Rigoli, "Harmonic Gauss maps", Pacific J. Math. 136:2 (1989), 261-282. MR 90e:53073 Zbl 0643.53040

[Klingenberg 1978] W. Klingenberg, A course in differential geometry, Graduate Text in Math. 51, Springer, New York, 1978. MR 57 \#13702 Zbl 0366.53001

[Kobayashi and Nomizu 1963] S. Kobayashi and K. Nomizu, Foundations of differential geometry, vol. 1, Wiley, New York, 1963. MR 97c:53001a Zbl 0119.37502

[O'Neill 1983] B. O'Neill, Semi-Riemannian geometry, with applications to relativity, Pure and Applied Mathematics 103, Academic Press, New York, 1983. MR 85f:53002 Zbl 0531.53051

[Vilms 1967] J. Vilms, “Connections on tangent bundles", J. Differential Geometry 1 (1967), 235243. MR 37 \#4742 Zbl 0162.53603

[Wu 1995] H. Wu, "Complete developable submanifolds in real and complex Euclidean spaces", Internat. J. Math. 6:3 (1995), 461-489. MR 96e:53083 Zbl 0839.53004

Received August 29, 2004.

\section{ROBERT J. FISHER}

DEPARTMENT OF MATHEMATICS

IDAHO STATE UNIVERSITY

POCATELLO, ID 83209-8085

UNITED STATES

fishrobe@isu.edu

H. TURNER LAQUER

DEPARTMENT OF MATHEMATICS

IDAHO STATE UNIVERSITY

POCATELLO, ID 83209-8085

UNITED STATES

laquerht@isu.edu 